\title{
Effective models for the multidimensional wave equation in heterogeneous media over long time and numerical homogenization
}

\author{
Assyr Abdulle \\ ANMC, Section de Mathématiques, \\ École Polytechnique Fédérale de Lausanne, \\ Station 8, CH-1015 Lausanne, Switzerland \\ assyr.abdulle@epfl.ch \\ Timothée Pouchon \\ ANMC, Section de Mathématiques, \\ École Polytechnique Fédérale de Lausanne, \\ Station 8, CH-1015 Lausanne, Switzerland \\ timothee.pouchon@epfl.ch
}

\begin{abstract}
A family of effective equations that capture the long time dispersive effects of wave propagation in heterogeneous media in an arbitrary large periodic spatial domain $\Omega \subset \mathbb{R}^{d}$ over long time is proposed and analyzed. For a wave equation with highly oscillatory periodic spatial tensors of characteristic length $\varepsilon$, we prove that the solution of any member of our family of effective equations are $\varepsilon$-close in the $\mathrm{L}^{\infty}\left(0, T^{\varepsilon}, \mathrm{L}^{2}(\Omega)\right)$ norm to the true oscillatory wave over a time interval of length $T^{\varepsilon}=\mathcal{O}\left(\varepsilon^{-2}\right)$. We show that the previously derived effective equation in [Dohnal, Lamacz, Schweizer, Multiscale Model. Simul., 2014] belongs to our family of effective equation. Moreover, while Bloch waves techniques were previously used, we show that asymptotic expansion techniques give an alternative way to derive such effective equations. An algorithm to compute the tensors involved in the dispersive equation and allowing for efficient numerical homogenization methods over long time is proposed.
\end{abstract}

Keywords: Homogenization; wave equation; effective equation; long time behavior; dispersive waves.

AMS Subject Classification: 35B27, 74Q10, 74Q15, 35L05

\section{Introduction}

The wave equation in heterogeneous media is used to model various engineering problems such as seismic inversion, medical imaging or the manufacture of composite materials. Given initial conditions, a source $f$ and a periodic tensor $a^{\varepsilon}$, we look for the wave displacement $u^{\varepsilon}$ such that

$$
\partial_{t}^{2} u^{\varepsilon}(t, x)-\nabla_{x} \cdot\left(a^{\varepsilon}(x)\left(\nabla_{x} u^{\varepsilon}(t, x)\right)=f(t, x) \quad \text { in }(0, T] \times \mathbb{R}^{d},\right.
$$

where $a^{\varepsilon}(x)=a\left(\frac{x}{\varepsilon}\right)=a(y)$ is $Y$-periodic in $y$ (a unit cell e.g., $Y=(-1 / 2,1 / 2)^{d}$ ). We assume for simplicity that $d \leq 3^{\text {a }}$. As the heterogeneities of the medium described by $a^{\varepsilon}$ arise at the microscopic scale $\mathcal{O}(\varepsilon)$, which is much smaller than the scale of interest $\mathcal{O}(1)$, standard numerical methods (finite difference method (FDM) or finite element method (FEM)) lead to a prohibitive computational cost as they require the resolution of the microscopic scale for the mesh size. Mathematically, the homogenization theory has been developed to deal with such problem (see Ref. 7, 18, 10, 21 for general theory, Ref. 9 for the wave equation). It provides the existence of a so called homogenized equation

$$
\partial_{t}^{2} u^{0}(t, x)-\sum_{i j=1}^{d} a_{i j}^{0} \partial_{i j}^{2} u^{0}(t, x)=f(t, x) \quad \text { in }(0, T] \times \mathbb{R}^{d},
$$

whose solution $u^{0}$ no longer oscillates at the microscopic scale and describes at short times $\mathcal{O}(1)$ the macroscopic behaviour $\mathcal{O}(1)$ of the wave $u^{\varepsilon}$. In this work, we assume the tensor $a^{\varepsilon}$ to

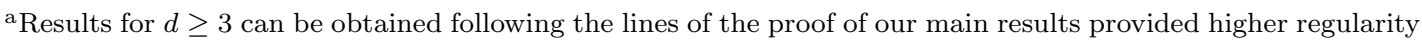
assumptions for $a(y)$. 
be periodic. In such case, the so-called homogenized tensor $a^{0} \in \mathbb{R}^{d \times d}$ in (1.2) can be computed explicitly via the solutions of $d$ cell problems. These cell problems are elliptic partial differential equations associated with $a$, with periodic boundary conditions.

However, it is known that at long times of order $\mathcal{O}\left(\varepsilon^{-2}\right)$, dispersion effects appear in the macroscopic behaviour of the wave $u^{\varepsilon}$, that are not captured by the homogenized solution $u^{0}$. In the literature, several papers have addressed this problem, with the purpose to define a higher order effective equation, i.e., an equation whose solution capture the dispersive effects of $u^{\varepsilon}$ over long times $\mathcal{O}\left(\varepsilon^{-2}\right)$. In all the results, this equation consists of (1.2) with some additional higher order constant differential operators. The challenge lies first in exhibiting the form of these operators, then defining the coefficients driving them and finally give an efficient algorithm to compute these coefficients. In Ref. 22, Santosa and Symes formally build an approximation of $u^{\varepsilon}$ (for $f=0$ ) over times $\mathcal{O}\left(\varepsilon^{-2}\right)$ that solves with a higher order remainder an equation of the form

$$
\partial_{t}^{2} u(t, x)-\sum_{i j=1}^{d} a_{i j}^{0} \partial_{i j}^{2} u(t, x)+\varepsilon^{2} \sum_{i j k l=1}^{d} c_{i j k l} \partial_{i j k l}^{4} u(t, x)=0 \quad \text { in }(0, T] \times \mathbb{R}^{d} .
$$

Unfortunately, $c$ being negative semidefinite, equation (1.3) is ill-posed. Nevertheless, numerical experiments show that a regularized approximation captures the desired dispersive effects of $u^{\varepsilon}$. Recently, several authors proposed a well-posed version of (1.3). The first rigorous result has been given by Lamacz in Ref. 19, in the one-dimensional case. An error estimate is proved over times $\mathcal{O}\left(\varepsilon^{-2}\right)$ between $u^{\varepsilon}$ (for $f=0$ ) and the solution of a Boussinesq type equation given by

$$
\partial_{t}^{2} u(t, x)-a^{0} \partial_{x}^{2} u(t, x)-\varepsilon^{2} b \partial_{x}^{2} \partial_{t}^{2} u(t, x)=0 \quad \text { in }(0, T] \times \mathbb{R},
$$

where the coefficient $b$ is computed via a cascade of 3 elliptic cell problems (including the cell problem necessary for $a^{0}$ ). In Ref. 4, the one-dimensional result from Ref. 19 was generalized and using the same technique it was proved that there exists a family of (well-posed) effective equations of the form (1.5) (for $d=1$ ) where the effective coefficients $b, c$ are computed with the help of a single cell problem (the same as to compute $a^{0}$ ).

The first rigorous error estimate over long times $\mathcal{O}\left(\varepsilon^{-2}\right)$ in the multi-dimensional case was proved by Dohnal, Lamacz and Schweizer in Ref. 14, 15. The (well-posed) effective equation is of the form (for $f=0$ )

$$
\partial_{t}^{2} u(t, x)-\sum_{i j=1}^{d} a_{i j}^{0} \partial_{i j}^{2} u(t, x)-\varepsilon^{2} \sum_{i j=1}^{d} b_{i j} \partial_{i j}^{2} \partial_{t}^{2} u(t, x)+\varepsilon^{2} \sum_{i j k l=1}^{d} c_{i j k l} \partial_{i j k l}^{4} u(t, x)=0,
$$

in $(0, T] \times \mathbb{R}^{d}$ where the tensors $b, c$ are computed via an algebraic decomposition of a 4 th order tensor, which is computed via a cascade of $d+\left(\begin{array}{c}d+1 \\ 2\end{array}\right)+\left(\begin{array}{c}d+2 \\ 3\end{array}\right)$ cell problems.

In this paper, we generalize the result from Ref. 4 to the multi-dimensional case, using the adaptation technique arising from asymptotic development introduced in Ref. 19. Our first main result is the definition of a family of well-posed effective equations of the same form as (1.5) and an error estimate that establish that any member of our family of effective equations is $\varepsilon$-close in the $\mathrm{L}^{\infty}\left(0, T^{\varepsilon} ; \mathrm{L}^{2}(\Omega)\right)$ norm to the true oscillatory wave over a time interval of length $T^{\varepsilon}=\mathcal{O}\left(\varepsilon^{-2}\right)$. The computation of the effective quantities involves only $d+\left(\begin{array}{c}d+1 \\ 2\end{array}\right)$ cell problems. The error estimate holds in an arbitrarily large periodic domain $\Omega \subset \mathbb{R}^{d}$, which makes our result comparable to the one from Ref. 14,15 (valid in the whole space $\mathbb{R}^{d}$ ). We also deal with more general settings than in Ref. 14, 15 as we allow for a source term in the equation and an initial speed. Finally, while the norm $\|u\|_{\mathrm{L}^{2}\left(\mathbb{R}^{d}\right)+\mathrm{L}^{\infty}\left(\mathbb{R}^{d}\right)}=\inf \left\{\left\|u_{1}\right\|_{\mathrm{L}^{2}\left(\mathbb{R}^{d}\right)}+\left\|u_{2}\right\|_{\mathrm{L}^{\infty}\left(\mathbb{R}^{d}\right)}: u=u_{1}+u_{2}\right\}$ on two Banach space was used in Ref. 14, 15, we obtain our error estimates in the stronger $L^{\infty}\left(0, T^{\varepsilon}, L^{2}(\Omega)\right)$ norm.

Error estimates between the oscillatory and the effective solutions can be obtained in different frameworks. In this paper (as in Ref. 4 and Ref. 19), the proof of the error estimate is 
done via the definition of an adaptation operator arising from asymptotic expansion, while in Ref. 14, 15 the expression of $u^{\varepsilon}$ in Bloch wave expansion is used (as it was formally introduced in Ref. 22). The interesting conclusion is that both techniques lead to the definition of valid effective equations. Our second result is to show that the effective equation from Ref. 14, 15 belongs to the family of effective equation that we define in this paper. We explicitly derive a correspondence between the solutions of the cell problems obtained with Bloch wave technique and the ones we obtain with asymptotic expansion. We note that this comparison has also been discussed independently in Ref. 5, with a focus on elliptic equations and an application to the wave equation.

Finally, we also derive an efficient computational algorithm to compute numerically the effective wave. We briefly discuss some related numerical strategies. In Ref. 16, Engquist, Holst and Runborg introduce a finite difference method (FDM) based on a regularized scheme for the ill-posed effective equation (1.3). The method is built in the framework of the heterogeneous multiscale method (HMM). In Ref. 6, this method is shown to capture the effective flux of the ill-posed equation given in Ref. 22 for the one-dimensional case. The finite heterogeneous method (FE-HMM) introduced in Ref. 1 has been modified in Ref. 2, 3 to approximate the effective model from Ref. 19. This method, the FE-HMM-L, has been fully analyzed in Ref. 4.

The paper is organized as follows. First, we introduce in Section 2 an error estimate to motivate the use of the asymptotic expansion that we then perform to obtain constraints on the effective coefficients. In Section 3, we state and prove our main result, the error estimate that leads to the definition of the family of effective equations. We then give in Section 4 a constructive method to obtain some elements of the family and describe an algorithm to compute the necessary effective quantities. In Section 5 , we relate our family of effective models to the effective model obtained in Ref. 14, 15 via Bloch wave expansion. Finally, in Section 6 we illustrate our theoretical findings through numerical experiments.

\section{Definitions and notations}

Let us denote the space of tensors of order $n$ by $\operatorname{Ten}^{n}\left(\mathbb{R}^{d}\right)$. A tensor $b \in \operatorname{Ten}^{n}\left(\mathbb{R}^{d}\right)$ is also denoted $\left\{b_{i_{1} \cdots i_{n}}\right\}$. In the whole text, we drop the notation of the sum symbol for the dot product between two tensors and use the convention that the repeated indices are summed, e.g., for $b \in \operatorname{Ten}^{3}\left(\mathbb{R}^{d}\right), c \in \operatorname{Ten}^{2}\left(\mathbb{R}^{d}\right), b_{i j k} c_{i j}=\sum_{i j=1}^{d} b_{i j k} c_{i j}$. Also, for a tensor of functions $v: \mathbb{R}^{d} \rightarrow \operatorname{Ten}^{n}\left(\mathbb{R}^{d}\right)$ the sum symbol is omitted in terms of the form $\partial_{x_{m}} v_{i_{1} \cdots m \cdots i_{n}}(x)=$ $\sum_{m=1}^{d} \partial_{x_{m}} v_{i_{1} \cdots m \cdots i_{n}}(x)$. Let us mention that the notation of the differentiation variable is sometimes omitted when there is no confusion. The subspace of $\operatorname{Ten}^{n}\left(\mathbb{R}^{d}\right)$ of symmetric tensors is denoted as $\operatorname{Sym}^{n}\left(\mathbb{R}^{d}\right)$, i.e., $b \in \operatorname{Sym}^{n}\left(\mathbb{R}^{d}\right)$ satisfies $b_{i_{1} \cdots i_{n}}=b_{i_{\sigma(1)} \cdots i_{\sigma(n)}}$ for any $\sigma \in S_{n}$ (permutations of order $n$ ). Finally, we introduce the symmetrization operator $S^{n}: \operatorname{Ten}^{n}\left(\mathbb{R}^{d}\right) \rightarrow$ $\operatorname{Sym}^{n}\left(\mathbb{R}^{d}\right)$, defined as

$$
\left(S^{n}(b)\right)_{i_{1} \cdots i_{n}}=\frac{1}{n !} \sum_{\sigma \in S_{n}} b_{i_{\sigma(1)} \cdots i_{\sigma(n)}} .
$$

In the text, $\left(S^{n}(b)\right)_{i_{1} \cdots i_{n}}$ is denoted as $S_{i_{1} \cdots i_{n}}^{n}\left\{b_{i_{1} \cdots i_{n}}\right\}$. Clearly, for $b \in \operatorname{Ten}^{n}\left(\mathbb{R}^{d}\right)$ we have $b_{i_{1} \cdots i_{n}} \partial_{i_{1} \cdots i_{n}}^{n}=S_{i_{1} \cdots i_{n}}^{n}\left\{b_{i_{1} \cdots i_{n}}\right\} \partial_{i_{1} \cdots i_{n}}^{n}$.

Let $\mathcal{O} \subset \mathbb{R}^{d}$ be an open hypercube and define the standard space of square integrable functions $\mathrm{L}^{2}(\mathcal{O})$ and the Sobolev space $\mathrm{H}^{k}(\mathcal{O})$. Equipped with their usual inner products, $\mathrm{L}^{2}(\mathcal{O})$ and $\mathrm{H}^{m}(\mathcal{O})$ are Hilbert spaces. The mean of an integrable function $v: \mathcal{O} \rightarrow \mathbb{R}$ is defined as $\langle v\rangle_{\mathcal{O}}=|\mathcal{O}|^{-1} \int_{\mathcal{O}} v(x) \mathrm{d} x$. We define the quotient space $\mathcal{L}^{2}(\mathcal{O})=\mathrm{L}^{2}(\mathcal{O}) / \mathbb{R}$ and denote by a bracket $[v]$ the equivalence class in $\mathcal{L}^{2}(\mathcal{O})$ of $v \in \mathrm{L}^{2}(\mathcal{O})$. Equipped with the inner product

$$
([v],[w])_{\mathcal{L}^{2}(\mathcal{O})}=\left(v-\langle v\rangle_{\mathcal{O}}, w-\langle w\rangle_{\mathcal{O}}\right)_{\mathrm{L}^{2}(\mathcal{O})}=(v, w)_{\mathrm{L}^{2}(\mathcal{O})}-|\mathcal{O}|\langle v\rangle_{\mathcal{O}}\langle w\rangle_{\mathcal{O}}
$$

where $v, w \in \mathrm{L}^{2}(\mathcal{O}), \mathcal{L}^{2}(\mathcal{O})$ is a Hilbert space. Let $\mathcal{C}_{\text {per }}^{\infty}(\mathcal{O})$ be the space of $\mathcal{O}$-periodic functions of $\mathcal{C}^{\infty}(\mathcal{O})$ and define the space $\mathrm{H}_{\text {per }}^{1}(\mathcal{O})$ as the closure of $\mathcal{C}_{\text {per }}^{\infty}(\mathcal{O})$ for the $\mathrm{H}^{1}$ norm. We define 
the quotient space $\mathcal{W}_{\text {per }}(\mathcal{O})=\mathrm{H}_{\text {per }}^{1}(\mathcal{O}) / \mathbb{R}$ and denote by a bold face letter $\boldsymbol{v}$ the elements of $\mathcal{W}_{\text {per }}(\mathcal{O})$. Equipped with the inner product

$$
(\boldsymbol{v}, \boldsymbol{w})_{\mathcal{W}_{\text {per }}(\mathcal{O})}=([v],[w])_{\mathcal{L}^{2}(\mathcal{O})}+\left(\partial_{k} v, \partial_{k} w\right)_{L^{2}(\mathcal{O})}, \quad \forall v \in \boldsymbol{v}, w \in \boldsymbol{w},
$$

and the induced norm $\|\boldsymbol{v}\|_{\mathcal{W}_{\text {per }}(\mathcal{O})}=\sqrt{(\boldsymbol{v}, \boldsymbol{v})_{\mathcal{W}_{\text {per }}(\mathcal{O})}}, \mathcal{W}_{\text {per }}(\mathcal{O})$ is a Hilbert space. Note that the $k$-th partial derivative of $\boldsymbol{v} \in \mathcal{W}_{\text {per }}(\mathcal{O})$ is simply $\partial_{k} \boldsymbol{v}=\partial_{k} v \in \mathrm{L}^{2}(\mathcal{O})$ for all $v \in \boldsymbol{v}$ and that thanks to the Poincaré-Wirtinger inequality, $\boldsymbol{v} \mapsto\|\nabla \boldsymbol{v}\|_{\mathrm{L}^{2}(\mathcal{O})}$ is also a norm on $\mathcal{W}_{\text {per }}(\mathcal{O})$, equivalent to $\|\cdot\|_{\mathcal{W}_{\text {per }}(\mathcal{O})}$. The dual space $\mathcal{W}_{\text {per }}^{*}(\mathcal{O})$ is characterized as follows : for $F \in \mathcal{W}_{\text {per }}^{*}(\mathcal{O})$, there exists $\left[f^{0}\right] \in \mathcal{L}^{2}(\mathcal{O}), f_{1}^{1}, \ldots, f_{d}^{1} \in \mathrm{L}^{2}(\mathcal{O})$ such that

$$
\langle F, \boldsymbol{v}\rangle_{\mathcal{W}_{\mathrm{per}}^{*}(\mathcal{O}), \mathcal{W}_{\mathrm{per}}(\mathcal{O})}=\left(\left[f^{0}\right], \boldsymbol{v}\right)_{\mathcal{L}^{2}(\mathcal{O})}+\left(f_{k}^{1}, \partial_{k} \boldsymbol{v}\right)_{\mathrm{L}^{2}(\mathcal{O})} \text {. }
$$

Furthermore, $\|F\|_{\mathcal{W}_{\text {per }}^{*}(\mathcal{O})}=\inf \left\{\left\|\left[f^{0}\right]\right\|_{\mathcal{L}^{2}(\mathcal{O})}+\left\|f^{1}\right\|_{L^{2}(\mathcal{O})}\right\}$, where the infimum is taken over all $\left[f^{0}\right] \in \mathcal{L}^{2}(\mathcal{O}), f^{1} \in \mathrm{L}^{2}(\mathcal{O})$ satisfying (1.7). From characterization (1.7), we verify that a functional of $\left[\mathrm{H}_{\text {per }}^{1}(\mathcal{O})\right]^{*}$ given by $w \mapsto\left(f^{0}, w\right)_{\mathrm{L}^{2}(\mathcal{O})}+\left(f_{k}^{1}, \partial_{k} w\right)_{\mathrm{L}^{2}(\mathcal{O})}$ for some $f^{0}, f_{1}^{1}, \ldots, f_{d}^{1} \in$ $\mathrm{L}^{2}(\mathcal{O})$, belongs to $\mathcal{W}_{\text {per }}^{*}(\mathcal{O})$ if and only if

$$
\left(f^{0}, 1\right)_{\mathrm{L}^{2}(\mathcal{O})}=0
$$

or equivalently $f^{0}$ has zero mean. Define $\mathrm{L}_{0}^{2}(\mathcal{O})$ (resp. $\mathrm{W}_{\text {per }}(\mathcal{O})$ ) as the set constituted with the zero mean representative of $\mathcal{L}^{2}(\mathcal{O})$ (resp. of $\mathcal{W}_{\text {per }}(\mathcal{O})$ ). Equipped with the standard $\mathrm{L}^{2}$ inner product (resp. $\left.\mathrm{H}^{1}\right), \mathrm{L}_{0}^{2}(\mathcal{O})$ is a Hilbert space (resp. $\mathrm{W}_{\text {per }}(\mathcal{O})$ ). Note that the following embeddings are dense $\mathrm{W}_{\text {per }}(\mathcal{O}) \subset \mathrm{L}_{0}^{2}(\mathcal{O}) \subset \mathrm{W}_{\text {per }}^{*}(\mathcal{O})$.

For a Banach space $X$ and $p \in[0, \infty), \mathrm{L}^{p}(0, T ; X)$ is the space of functions $v:[0, T] \rightarrow X$ such that $\|v\|_{\mathrm{L}^{p}(0, T ; X)}=\left(\int_{0}^{T}\|v(t)\|_{X}^{p} \mathrm{~d} t\right)^{1 / p}<\infty$. The definition is similar for $p=\infty$, with the $\mathrm{L}^{\infty}$ norm in time. To simplify the notation we will often use the shorthand notation $\|\cdot\|_{\mathrm{L}^{p}},\|\cdot\|_{\mathrm{L}^{p}(X)},(\cdot, \cdot)_{\mathcal{O}}$ instead of $\|\cdot\|_{\mathrm{L}^{p}(\mathcal{O})},\|\cdot\|_{\mathrm{L}^{p}(0, T ; X)}$ and $(\cdot, \cdot)_{\mathrm{L}^{2}(\mathcal{O})}$ respectively.

\section{The wave equation in a heterogeneous medium}

Let us now introduce precisely the settings for equation (1.1) in an arbitrarily large periodic domain. Let $\Omega, Y \in \mathbb{R}^{d}$ be open hypercubes such that $\Omega$ is a union of cells of volume $\varepsilon|Y|$, as in Figure 1. We assume that $a$ is a $Y$-periodic $d \times d$ tensor and hence $a\left(\frac{x}{\varepsilon}\right)$ is $\Omega$-periodic ( $a$ is extended by periodicity).

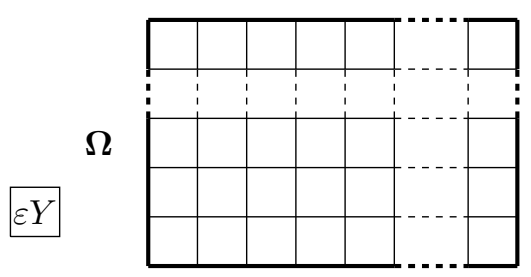

Fig. 1. The hypercube $\Omega$ is assumed to be a union of unit cells of volume $\varepsilon|Y|$ (in the picture $d=2$ ).

For $T^{\varepsilon}=\varepsilon^{-2} T$, we consider the wave equation : find $u^{\varepsilon}:\left[0, T^{\varepsilon}\right] \times \Omega \rightarrow \mathbb{R}$ such that

$$
\begin{array}{ll}
\partial_{t}^{2} u^{\varepsilon}(t, x)-\nabla_{x} \cdot\left(a\left(\frac{x}{\varepsilon}\right) \nabla_{x} u^{\varepsilon}(t, x)\right)=f(t, x) & \text { in }\left(0, T^{\varepsilon}\right] \times \Omega, \\
x \mapsto u^{\varepsilon}(t, x) \Omega \text {-periodic } & \text { in }\left[0, T^{\varepsilon}\right], \\
u^{\varepsilon}(0, x)=g^{0}(x), \quad \partial_{t} u^{\varepsilon}(0, x)=g^{1}(x) & \text { in } \Omega,
\end{array}
$$

where $g^{0}, g^{1}$ are given initial conditions and $f$ is a source. The following notation is used for the differential operator $\mathcal{A}^{\varepsilon}=-\nabla_{x} \cdot\left(a\left(\frac{x}{\varepsilon}\right) \nabla_{x}(\cdot)\right)$. We assume that $a \in\left[\mathrm{L}^{\infty}(Y)\right]^{d \times d}$ is symmetric, uniformly elliptic and bounded, i.e. there exists $\lambda, \Lambda>0$ such that

$$
\lambda|\xi|^{2} \leq a(y) \xi \cdot \xi \leq \Lambda|\xi|^{2} \text { for a.e. } y \in Y \quad \forall \xi \in \mathbb{R}^{d} \text {. }
$$


For the well-posedness of problem (1.9), we refer to Lions and Magenes in Ref. 20. A detailed proof may be found in Ref. 17. If $g^{0} \in \mathrm{W}_{\text {per }}(\Omega), g^{1} \in \mathrm{L}_{0}^{2}(\Omega), f \in \mathrm{L}^{2}\left(0, T^{\varepsilon} ; \mathrm{L}^{2}(\Omega)\right)$ then there exists a unique weak solution $u^{\varepsilon} \in \mathrm{L}^{2}\left(0, T^{\varepsilon} ; \mathrm{W}_{\text {per }}(\Omega)\right)$ with $\partial_{t} u^{\varepsilon} \in \mathrm{L}^{2}\left(0, T^{\varepsilon} ; \mathrm{L}_{0}^{2}(\Omega)\right)$ and $\partial_{t}^{2} u^{\varepsilon} \in$ $\mathrm{L}^{2}\left(0, T^{\varepsilon} ; \mathrm{W}_{\text {per }}^{*}(\Omega)\right)$. We note that $u^{\varepsilon}$ is proved to be even more regular, $u^{\varepsilon} \in \mathcal{C}^{0}\left(\left[0, T^{\varepsilon}\right] ; \mathrm{W}_{\text {per }}(\Omega)\right)$ and $\partial_{t} u^{\varepsilon} \in \mathcal{C}^{0}\left(\left[0, T^{\varepsilon}\right] ; \mathrm{L}_{0}^{2}(\Omega)\right)$.

\section{Effective coefficients via asymptotic expansion}

Asymptotic expansion is a formal technique systematically used in homogenization theory to derive effective equations (see Ref. $7,18,10$ ). In this section, we explain how asymptotic expansion is used to define an adaptation operator and prove a rigorous estimate of $\| u^{\varepsilon}-$ $\tilde{u} \|_{\mathrm{L}^{2}\left(0, T^{\varepsilon} ; \mathrm{L}^{2}(\Omega)\right)}, \tilde{u}$ being an effective solution. First, we prove an energy estimate that is central in the proof of the error estimate. Second, we proceed to the asymptotic expansion and obtain constraints for the definition of the tensors of the effective equations.

\subsection{An error estimate to motivate asymptotic expansion}

Consider $\mathcal{B}^{\varepsilon} \tilde{u}$, an adaptation of the effective solution $\tilde{u}$ (as defined in (3.7)). The following abstract lemma gives a general error estimate that clarifies the requirements needed $\boldsymbol{B}^{\varepsilon}$ for the adaptation to be a good approximation of $u^{\varepsilon}$ on a long time interval.

Lemma 2.1. Let $T^{\varepsilon}=\varepsilon^{-\alpha}, \alpha \geq 0$. Assume that $\tilde{u}:\left[0, T^{\varepsilon}\right] \times \Omega \rightarrow \mathbb{R}$ is a function such that $\tilde{u}(0)=g^{0}, \partial_{t} \tilde{u}(0)=g^{1}$. Furthermore, assume that $\mathcal{B}^{\varepsilon}$ is an operator such that $\mathcal{B}^{\varepsilon} \tilde{u}$ belongs to $\mathrm{L}^{\infty}\left(0, T^{\varepsilon} ; \mathcal{W}_{\text {per }}(\Omega)\right)$, with $\partial_{t} \mathcal{B}^{\varepsilon} \tilde{u} \in \mathrm{L}^{\infty}\left(0, T^{\varepsilon} ; \mathcal{L}^{2}(\Omega)\right), \partial_{t}^{2} \mathcal{B}^{\varepsilon} \tilde{u} \in \mathrm{L}^{2}\left(0, T^{\varepsilon} ; \mathcal{W}_{\text {per }}^{*}(\Omega)\right)$ and $\partial_{t} \mathcal{B}^{\varepsilon} \tilde{u}(0)=\mathcal{B}^{\varepsilon} g^{1}$. Finally, assume that it holds

$$
\left(\partial_{t}^{2}+\mathcal{A}^{\varepsilon}\right)\left(\mathcal{B}^{\varepsilon} \tilde{u}-\left[u^{\varepsilon}\right]\right)(t)=\boldsymbol{r}^{\varepsilon}(t) \text { in } \mathcal{W}_{\mathrm{per}}^{*}(\Omega) \text { for a.e. } t \in\left[0, T^{\varepsilon}\right],
$$

where $\boldsymbol{r}^{\varepsilon} \in \mathrm{L}^{\infty}\left(0, T^{\varepsilon} ; \mathcal{W}_{\text {per }}^{*}(\Omega)\right)$. Then, the following error estimate holds

$$
\begin{gathered}
\left\|\mathcal{B}^{\varepsilon} \tilde{u}-\left[u^{\varepsilon}\right]\right\|_{L^{\infty}\left(0, T^{\varepsilon} ; \mathcal{L}^{2}(\Omega)\right)} \leq C\left(\left\|\mathcal{B}^{\varepsilon} g^{1}-\left[g^{1}\right]\right\|_{\mathcal{W}_{\mathrm{per}}^{*}(\Omega)}+\left\|\boldsymbol{B}^{\varepsilon} g^{0}-\left[g^{0}\right]\right\|_{\mathcal{L}^{2}(\Omega)}\right. \\
\left.+\varepsilon^{-\alpha}\left\|\boldsymbol{r}^{\varepsilon}\right\|_{L^{\infty}\left(0, T^{\varepsilon} ; \mathcal{W}_{\mathrm{per}}^{*}(\Omega)\right)}\right),
\end{gathered}
$$

where $C$ depends only on $\lambda, \Lambda$ and $T$.

Proof. To simplify the notations we note $\langle\cdot, \cdot\rangle$ instead of $\langle\cdot, \cdot\rangle_{\mathcal{W}_{\mathrm{per}}^{*},}, \mathcal{W}_{\mathrm{per}}$ and $\mathcal{A}=\mathcal{A}^{\varepsilon}$. Thanks to Lax-Milgram theorem, define the inverse of $\mathcal{A}$, noted $\mathcal{A}^{-1}$. Using the properties of $a$, we can show that $\mathcal{A}^{-1}$ is self-adjoint, elliptic $\left(\left\langle F, \mathcal{A}^{-1} F\right\rangle \geq \Lambda^{-1}\|F\|_{\mathcal{W}_{\text {per }}^{*}}^{2}\right)$ and bounded $\left(\left\|\mathcal{A}^{-1}\right\| \leq \lambda^{-1}\right)$. Let $\boldsymbol{\eta}=\mathcal{B}^{\varepsilon} \tilde{u}-\left[u^{\varepsilon}\right]$. Using (2.1) with the test function $\boldsymbol{w}=\mathcal{A}^{-1} \partial_{t} \boldsymbol{\eta}(t)$, we obtain for a.e. $t \in\left[0, T^{\varepsilon}\right]$

$$
\frac{1}{2} \frac{\mathrm{d}}{\mathrm{d} t}\left(\left\langle\partial_{t} \boldsymbol{\eta}(t), \mathcal{A}^{-1} \partial_{t} \boldsymbol{\eta}(t)\right\rangle+\|\boldsymbol{\eta}(t)\|_{\mathcal{L}^{2}}^{2}\right)=\left\langle\boldsymbol{r}^{\varepsilon}(t), \mathcal{A}^{-1} \partial_{t} \boldsymbol{\eta}(t)\right\rangle .
$$

Setting $E \boldsymbol{\eta}(t)=\left\langle\partial_{t} \boldsymbol{\eta}(t), \mathcal{A}^{-1} \partial_{t} \boldsymbol{\eta}(t)\right\rangle+\|\boldsymbol{\eta}(t)\|_{\mathcal{L}^{2}}^{2}$, we integrate (2.3) over $[0, \xi]$ to get

$$
E \boldsymbol{\eta}(\xi)=E \boldsymbol{\eta}(0)+2 \int_{0}^{\xi}\left\langle\boldsymbol{r}^{\varepsilon}(t), \mathcal{A}^{-1} \partial_{t} \boldsymbol{\eta}(t)\right\rangle \mathrm{d} t \quad \forall \xi \in\left[0, T^{\varepsilon}\right] .
$$

Using Hölder and Young inequalities and the bounededness of $\mathcal{A}^{-1}$, we obtain

$$
E \boldsymbol{\eta}(\xi) \leq E \boldsymbol{\eta}(0)+2 \Lambda / \lambda^{2}\left\|\boldsymbol{r}^{\varepsilon}\right\|_{\mathrm{L}^{1}\left(\mathcal{W}_{\mathrm{per}}^{*}\right)}^{2}+1 /(2 \Lambda)\left\|\partial_{t} \boldsymbol{\eta}\right\|_{\mathrm{L}^{\infty}\left(\mathcal{W}_{\mathrm{per}}^{*}\right)}^{2} .
$$

Using the ellipticity of $\mathcal{A}^{-1}$ we have $1 / \Lambda\left\|\partial_{t} \boldsymbol{\eta}(\xi)\right\|_{\mathcal{W}_{\mathrm{per}}^{*}}^{2} \leq E \boldsymbol{\eta}(\xi)$, hence, taking the $\mathrm{L}^{\infty}$ norm with respect to $\xi$, we obtain $1 /(2 \Lambda)\left\|\partial_{t} \boldsymbol{\eta}\right\|_{\mathrm{L}^{\infty}\left(\mathcal{W}_{\mathrm{per}}^{*}\right)}^{2} \leq E \boldsymbol{\eta}(0)+2 \Lambda / \lambda^{2}\left\|\boldsymbol{r}^{\varepsilon}\right\|_{\mathrm{L}^{1}\left(\mathcal{W}_{\mathrm{per}}^{*}\right)}^{2}$. As $\|\boldsymbol{\eta}(\xi)\|_{\mathrm{L}^{\infty}\left(\mathcal{L}^{2}\right)}^{2} \leq$ $E \boldsymbol{\eta}(\xi)$, estimate (2.4) and the boundedness of $\mathcal{A}^{-1}$ gives

$$
\left.\|\boldsymbol{\eta}\|_{L^{\infty}\left(\mathcal{L}^{2}\right)}^{2} \leq 2 / \lambda\left\|\partial_{t} \boldsymbol{\eta}(0)\right\|_{\mathcal{W}_{\mathrm{Per}}^{*}}^{2}+2\|\boldsymbol{\eta}(0)\|_{\mathcal{L}^{2}}^{2}+4 \Lambda / \lambda^{2}\left\|\boldsymbol{r}^{\varepsilon}\right\|_{\mathrm{L}^{1}\left(\mathcal{W}_{\mathrm{per}}^{*}\right.}^{2}\right) .
$$


Thanks to Hölder inequality we have $\left\|\boldsymbol{r}^{\varepsilon}\right\|_{\mathrm{L}^{1}\left(\mathcal{W}_{\mathrm{per}}^{*}\right)} \leq T \varepsilon^{-\alpha}\left\|\boldsymbol{r}^{\varepsilon}\right\|_{\mathrm{L}^{\infty}\left(\mathcal{W}_{\mathrm{per}}^{*}\right)}$. Finally, we have $\partial_{t} \boldsymbol{\eta}(0)=\mathcal{B}^{\varepsilon} g^{1}-\left[g^{1}\right], \boldsymbol{\eta}(0)=\mathcal{B}^{\varepsilon} g^{0}-\left[g^{0}\right]$. The proof of the lemma is complete.

Let us explain how Lemma 2.1 and the asymptotic expansion (2.9) lead to an error estimate for effective equations. On a time interval $\left[0, T^{\varepsilon}\right], T^{\varepsilon}=\varepsilon^{-\alpha} T$, let $\mathcal{B}^{\varepsilon}$ be an adaptation of the effective solution $\tilde{u}$ of the form $\mathcal{B}^{\varepsilon} \tilde{u}=[\tilde{u}]+\mathcal{C}^{\varepsilon} \tilde{u}$, where $\left\|\mathcal{C}^{\varepsilon} \tilde{u}\right\|_{\mathrm{L}^{\infty}\left(\mathcal{L}^{2}\right)} \leq C \varepsilon$. Then, since $\tilde{u}(0)=g^{0}, \partial_{t} \tilde{u}(0)=g^{1}$, the error estimate $(2.2)$ becomes

$$
\left\|\mathcal{B}^{\varepsilon} \tilde{u}-\left[u^{\varepsilon}\right]\right\|_{L^{\infty}\left(0, T^{\varepsilon} ; \mathcal{L}^{2}(\Omega)\right)} \leq C \varepsilon+\varepsilon^{-\alpha}\left\|\boldsymbol{r}^{\varepsilon}\right\|_{\mathrm{L}^{\infty}\left(0, T^{\varepsilon} ; \mathcal{W}_{\mathrm{per}}^{*}(\Omega)\right)} .
$$

As we also have $\left\|[\tilde{u}]-\mathcal{B}^{\varepsilon} \tilde{u}\right\|_{L^{\infty}\left(0, T^{\varepsilon} ; \mathcal{L}^{2}(\Omega)\right)} \leq C \varepsilon$, we obtain via the triangle inequality (and using $\left.\langle\tilde{u}\rangle_{\Omega}=\left\langle u^{\varepsilon}\right\rangle_{\Omega}\right)$,

$$
\left\|u^{\varepsilon}-\tilde{u}\right\|_{L^{\infty}\left(0, T^{\varepsilon} ; \mathrm{L}^{2}(\Omega)\right)}=\left\|\left[u^{\varepsilon}-\tilde{u}\right]\right\|_{\mathrm{L}^{\infty}\left(0, T^{\varepsilon} ; \mathcal{L}^{2}(\Omega)\right)} \leq C \varepsilon+\varepsilon^{-\alpha}\left\|\boldsymbol{r}^{\varepsilon}\right\|_{\mathrm{L}^{\infty}\left(0, T^{\varepsilon} ; \mathcal{W}_{\mathrm{per}}^{*}(\Omega)\right)} .
$$

Estimate (2.6) implies the following : if a function $\tilde{u}$ is such that we can define an $\Omega$-periodic adaptation $\mathcal{B}^{\varepsilon} \tilde{u}$ satisfying $\left(\partial_{t}^{2}+\mathcal{A}^{\varepsilon}\right)\left(\mathcal{B}^{\varepsilon} \tilde{u}-\left[u^{\varepsilon}\right]\right)=\mathcal{O}\left(\varepsilon^{\gamma}\right)$, where $\gamma>\alpha$, then $\tilde{u}$ approximates $u^{\varepsilon}$ up to times $\varepsilon^{-\alpha} T$ with accuracy $\mathcal{O}\left(\varepsilon^{\gamma-\alpha}\right)$. The construction of $\mathcal{B}^{\varepsilon} \tilde{u}$ is done via asymptotic expansion as explained in Section 2.2.

Note that the presence of the $\mathrm{L}^{\infty}$ norm in time for the term $\boldsymbol{r}^{\varepsilon}$ in estimate (2.2) (and (2.6)) is "responsible" for the $\varepsilon^{-\alpha}$ factor. As we will see, in practice, asymptotic development leads to a remainder in (2.1) of the form $\boldsymbol{r}^{\varepsilon}=\boldsymbol{\mathcal { R }}^{\varepsilon} \tilde{u}$, where $\left\|\boldsymbol{\mathcal { R }}^{\varepsilon} \tilde{u}\right\|_{\mathrm{L}^{\infty}\left(\mathcal{W}_{\mathrm{per}}^{*}\right)} \leq C \varepsilon^{\gamma} \sum_{k=0,2}\left\|\partial_{t}^{k} \tilde{u}\right\|_{\mathrm{L}^{\infty}\left(\mathrm{H}^{s} k\right)}$. As the energy estimate for hyperbolic problems gives a bound for $\left\|\partial_{t}^{k} \tilde{u}\right\|_{\mathrm{L}^{\infty}\left(\mathrm{H}^{s}\right)}$, the $\mathrm{L}^{\infty}$ norm is the "right" quantification in time for the remainder $\boldsymbol{r}^{\varepsilon}$.

\subsection{Asymptotic expansion and constraints on the effective coefficients}

We now perform the asymptotic expansion. All the computations are done formally, i.e., we assume as much regularity as required. The rigorous result with its detailed proof is presented in the next section.

We are looking for an effective solution on a time interval $\left[0, T^{\varepsilon}\right], T^{\varepsilon}=\varepsilon^{-2} T$. As discussed in the previous section, we thus need to construct an adaptation $\mathcal{B}^{\varepsilon} \tilde{u}(t)$ such that $\left(\partial_{t}^{2}+\mathcal{A}^{\varepsilon}\right)\left(\mathcal{B}^{\varepsilon} \tilde{u}-\right.$ $\left.\left[u^{\varepsilon}\right]\right)(t)=\mathcal{O}\left(\varepsilon^{3}\right)$ for a.e. $t$. We first construct $\mathcal{B}^{\varepsilon} \tilde{u}(t) \in \mathrm{H}_{\mathrm{per}}^{1}(\Omega)$, such that $\left(\partial_{t}^{2}+\mathcal{A}^{\varepsilon}\right)\left(\mathcal{B}^{\varepsilon} \tilde{u}-\right.$ $\left.u^{\varepsilon}\right)(t)=\mathcal{O}\left(\varepsilon^{3}\right)$ and we will then set $\mathcal{B}^{\varepsilon} \tilde{u}=\left[\mathcal{B}^{\varepsilon} \tilde{u}\right]$ in $\mathcal{W}_{\mathrm{per}}^{*}(\Omega)$. The construction of $\mathcal{B}^{\varepsilon} \tilde{u}$ leads to cell problems that are elliptic PDEs with periodic boundary conditions, whose solutions are called correctors. We will see that the well-posedness of these cell problems constraint the definition of the effective tensors.

First, we introduce the effective solution $\tilde{u}$. Referring to Ref. 15, 14, 4, we make the ansatz that the effective equation is of the form

$$
\begin{array}{ll}
\partial_{t}^{2} \tilde{u}-a_{i j}^{0} \partial_{i j}^{2} \tilde{u}+\varepsilon^{2}\left(a_{i j k l}^{2} \partial_{i j k l}^{4} \tilde{u}-b_{i j}^{0} \partial_{i j}^{2} \partial_{t}^{2} \tilde{u}\right)=f & \text { in }\left(0, T^{\varepsilon}\right] \times \Omega, \\
x \mapsto \tilde{u}(t, x) \Omega \text {-periodic } & \text { in }\left[0, T^{\varepsilon}\right], \\
\tilde{u}(0, x)=g^{0}(x), \quad \partial_{t} \tilde{u}(0, x)=g^{1}(x) & \text { in } \Omega,
\end{array}
$$

where $b^{0} \in \operatorname{Ten}^{2}\left(\mathbb{R}^{d}\right), a^{2} \in \operatorname{Ten}^{4}\left(\mathbb{R}^{d}\right)$ are coefficients to determine and $a^{0} \in \operatorname{Sym}^{2}\left(\mathbb{R}^{d}\right)$ is the homogeneous tensor defined by Ref. $7,18,10$

$$
a_{i j}^{0}=\left\langle e_{i}^{T} a\left(\nabla \chi_{j}+e_{j}\right)\right\rangle_{Y}
$$

where $\chi_{j}$ belongs to the class of solutions of (2.12). Next, we make a second ansatz : the adaptation of $\tilde{u}$ is of the form

$$
\mathcal{B}^{\varepsilon} \tilde{u}(t, x)=\tilde{u}(t, x)+\varepsilon u^{1}\left(t, x, \frac{x}{\varepsilon}\right)+\varepsilon^{2} u^{2}\left(t, x, \frac{x}{\varepsilon}\right)+\varepsilon^{3} u^{3}\left(t, x, \frac{x}{\varepsilon}\right)+\varepsilon^{4} u^{4}\left(t, x, \frac{x}{\varepsilon}\right),
$$

where the $u^{i}(t, x, y)$ are $\Omega$-periodic in $x$ and $Y$-periodic in $y$. We introduce the differential operators

$$
\begin{gathered}
\mathcal{A}^{0}=-\nabla_{y} \cdot\left(a(y) \nabla_{y} \cdot\right), \quad \mathcal{A}^{1}=-\nabla_{y} \cdot\left(a(y) \nabla_{x} \cdot\right)-\nabla_{x} \cdot\left(a(y) \nabla_{y} \cdot\right), \\
\mathcal{A}^{2}=-\nabla_{x} \cdot\left(a(y) \nabla_{x} \cdot\right),
\end{gathered}
$$


so that for $\psi(x, y)$ smooth enough, using the chain rule, we have $\mathcal{A}^{\varepsilon} \psi\left(x, \frac{x}{\varepsilon}\right)=\left(\varepsilon^{-2} \mathcal{A}^{0}+\right.$ $\left.\varepsilon^{-1} \mathcal{A}^{1}+\mathcal{A}^{2}\right) \psi\left(x, \frac{x}{\varepsilon}\right)$. We fix a $t \in\left[0, T^{\varepsilon}\right]$ and using equations (1.9), (2.7) and ansatz (2.9), we compute

$$
\begin{aligned}
\left(\partial_{t}^{2}+\mathcal{A}^{\varepsilon}\right)\left(\mathcal{B}^{\varepsilon} \tilde{u}-u^{\varepsilon}\right)(t, x)=\partial_{t}^{2} \mathcal{B}^{\varepsilon} \tilde{u}(t, x)+\mathcal{A}^{\varepsilon} \mathcal{B}^{\varepsilon} \tilde{u}(t, x)-f(t, x) & ) \\
= & \varepsilon^{-1}\left(\mathcal{A}^{0} u^{1}+\mathcal{A}^{1} \tilde{u}\right. \\
& +\varepsilon^{0}\left(\mathcal{A}^{0} u^{2}+\mathcal{A}^{1} u^{1}+\mathcal{A}^{2} \tilde{u}+a_{i j}^{0} \partial_{i j}^{2} \tilde{u}\right. \\
& +\varepsilon^{1}\left(\partial_{t}^{2} u^{1}+\mathcal{A}^{0} u^{3}+\mathcal{A}^{1} u^{2}\right. \\
& +\varepsilon^{2}\left(\partial_{t}^{2} u^{2}+\mathcal{A}^{0} u^{4}+\mathcal{A}^{1} u^{3}+\mathcal{A}^{2} u^{2}-a_{i j k l}^{2} \partial_{i j k l}^{4} \tilde{u}+b_{i j}^{0} \partial_{i j}^{2} \partial_{t}^{2} \tilde{u}\right) \\
& +\mathcal{O}\left(\varepsilon^{3}\right),
\end{aligned}
$$

where the $u^{i}$ are evaluated at $\left(t, x, y=\frac{x}{\varepsilon}\right)$. We now define successively $u^{1}$ to $u^{4}$ so that the terms of order $\mathcal{O}\left(\varepsilon^{-2}\right)$ to $\mathcal{O}\left(\varepsilon^{2}\right)$ in $(2.10)$ cancel. At order $\mathcal{O}\left(\varepsilon^{-1}\right)$, we obtain the equation $\mathcal{A}^{0} u^{1}+\mathcal{A}^{1} \tilde{u}=0$ which reads

$$
-\nabla_{y} \cdot\left(a(y)\left(\nabla_{y} u^{1}(t, x, y)+\nabla_{x} \tilde{u}(t, x)\right)\right)=0 .
$$

We can show that any solution of this elliptic equation is of the form $\chi_{i}(y) \partial_{i} \tilde{u}(t, x)+c_{1}(t, x)$, where $c_{1}$ is a function independent of $y$ and for all $1 \leq i \leq d, \chi_{i}$ is $Y$-periodic and solves the cell problem

$$
-\nabla_{y} \cdot\left(a\left(\nabla_{y} \chi_{i}+e_{i}\right)\right)=0 \text { in } Y .
$$

For simplicity, we choose $u_{1}(t, x, y)=\chi_{i}(y) \partial_{i} \tilde{u}(t, x)$. Consider now the $\mathcal{O}(1)$ order term in (2.10), which reads now

$$
-\nabla_{y} \cdot\left(a(y) \nabla_{y} u^{2}(t, x, y)\right)=\left(\nabla_{y} \cdot\left(a(y) e_{i} \chi_{j}(y)\right)+e_{i}^{T} a(y)\left(\nabla_{y} \chi_{j}(y)+e_{j}\right)-a_{i j}^{0}\right) \partial_{i j}^{2} \tilde{u}(t, x) .
$$

The solution is given by $u^{2}(t, x, y)=\tilde{\theta}_{i j}(y) \partial_{i j}^{2} \tilde{u}(t, x)+c_{2}(t, x)$, where for $1 \leq i, j \leq d \tilde{\theta}_{i j}$ is $Y$-periodic and solves the cell problem

$$
-\nabla_{y} \cdot\left(a \nabla_{y} \tilde{\theta}_{i j}\right)=\nabla_{y} \cdot\left(a e_{i} \chi_{j}\right)+e_{i}^{T} a \nabla_{y} \chi_{j}+a_{i j}-a_{i j}^{0} \quad \text { in } Y .
$$

Once again, we let $c_{2}=0$ for simplicity. We note here that for sufficiently smooth $\tilde{u}, u^{2}$ can also be written as $\theta_{i j}(y) \partial_{i j}^{2} \tilde{u}(t, x)$, where $\theta_{i j}=\frac{1}{2}\left(\tilde{\theta}_{i j}+\tilde{\theta}_{j i}\right)=S_{i j}^{2}\left\{\tilde{\theta}_{i j}\right\}$ is the symmetrization (1.6) of $\tilde{\theta}_{i j}$ and solves the cell problem

$$
-\nabla_{y} \cdot\left(a \nabla_{y} \theta_{i j}\right)=S_{i j}^{2}\left\{\nabla_{y} \cdot\left(a e_{i} \chi_{j}\right)+e_{i}^{T} a \nabla_{y} \chi_{j}+a_{i j}-a_{i j}^{0}\right\} \quad \text { in } Y .
$$

The advantage of the second form of $u^{2}$ is that there are only $\left(\begin{array}{c}d+1 \\ 2\end{array}\right)$ cell problems describing $\left\{\theta_{i j}\right\}$ compared to the $d^{2}$ for $\left\{\tilde{\theta}_{i j}\right\}$. Before canceling the $\mathcal{O}(\varepsilon)$ and $\mathcal{O}\left(\varepsilon^{2}\right)$ order terms, we rewrite (2.10) taking into account the definition of $u^{1}$ and $u^{2}$. Using (2.7), we have

$$
\begin{aligned}
& \partial_{t}^{2} u^{1}=\chi_{i} \partial_{i} \partial_{t}^{2} \tilde{u}=\chi_{i} \partial_{i} f+a_{i j}^{0} \chi_{k} \partial_{i j k}^{3} \tilde{u}+\mathcal{O}\left(\varepsilon^{2}\right), \\
& \partial_{t}^{2} u^{2}=\theta_{i j} \partial_{i j}^{2} \partial_{t}^{2} \tilde{u}=\theta_{i j} \partial_{i j}^{2} f+a_{i j}^{0} \theta_{k l} \partial_{i j k l}^{4} \tilde{u}+\mathcal{O}(\varepsilon), \\
& b^{0} \partial_{x}^{2} \partial_{t}^{2} \tilde{u}=b_{i j}^{0} \partial_{i j}^{2} f+a_{i j}^{0} b_{k l}^{0} \partial_{i j k l}^{4} \tilde{u}+\mathcal{O}(\varepsilon),
\end{aligned}
$$

hence (2.10) reads

$$
\begin{aligned}
\left(\partial_{t}^{2}+\mathcal{A}^{\varepsilon}\right)\left(\mathcal{B}^{\varepsilon} \tilde{u}-u^{\varepsilon}\right)(t, x)= & \varepsilon^{1}\left(\mathcal{A}^{0} u^{3}+\mathcal{A}^{1} u^{2}+a_{i j}^{0} \chi_{k} \partial_{i j k}^{3} \tilde{u}\right) \\
& +\varepsilon^{2}\left(\mathcal{A}^{0} u^{4}+\mathcal{A}^{1} u^{3}+\mathcal{A}^{2} u^{2}+\left(a_{i j}^{0}\left(b_{k l}^{0}+\theta_{k l}\right)-a_{i j k l}^{2}\right) \partial_{i j k l}^{4} \tilde{u}\right) \\
& +\varepsilon^{1} \chi_{i} \partial_{i} f+\varepsilon^{2}\left(b_{i j}^{0}+\theta_{i j}\right) \partial_{i j}^{2} f+\mathcal{O}\left(\varepsilon^{3}\right) .
\end{aligned}
$$

Let us first assume that $f=0$. To cancel the $\mathcal{O}(\varepsilon)$ and $\mathcal{O}\left(\varepsilon^{2}\right)$ order terms in (2.11), we can set $u^{3}(t, x, y)=\kappa_{i j k}(y) \partial_{i j k}^{3} \tilde{u}$, and $u^{4}(t, x, y)=\rho_{i j k l}(y) \partial_{i j k l}^{4} \tilde{u}$, where $\kappa_{i j k}$ and $\rho_{i j k l}$ are the solutions of cell problems obtained in a similar manner as for $\chi_{i}$ and $\theta_{i j}$. As previously, in order to minimize the number of cell problems, we use the symmetrization operators $S^{3}$ and 
$S^{4}$. In summary, we obtain the following cell problems : for $1 \leq i, j, k, l \leq d$, find $Y$-periodic functions $\chi_{i}, \theta_{i j}, \kappa_{i j k}, \rho_{i j k l}$ such that

$$
\begin{aligned}
& \varepsilon^{-1}:\left(a \nabla_{y} \chi_{i}, \nabla_{y} w\right)_{Y}=-\left(a e_{i}, \nabla_{y} w\right)_{Y}, \\
& \varepsilon^{0}:\left(a \nabla_{y} \theta_{i j}, \nabla_{y} w\right)_{Y}=S_{i j}^{2}\left\{-\left(a e_{i} \chi_{j}, \nabla_{y} w\right)_{Y}+\left(a\left(\nabla_{y} \chi_{j}+e_{j}\right)-a^{0} e_{j}, e_{i} w\right)_{Y}\right\} \text {, } \\
& \varepsilon^{1}:\left(a \nabla_{y} \kappa_{i j k}, \nabla_{y} w\right)_{Y}=S_{i j k}^{3}\left\{-\left(a e_{i} \theta_{j k}, \nabla_{y} w\right)_{Y}\right. \\
& \left.+\left(a\left(\nabla_{y} \theta_{j k}+e_{j} \chi_{k}\right)-a^{0} e_{j} \chi_{k}, e_{i} w\right)_{Y}\right\}, \\
& \varepsilon^{2}:\left(a \nabla_{y} \rho_{i j k l}, \nabla_{y} w\right)_{Y}=S_{i j k l}^{4}\left\{-\left(a e_{i} \kappa_{j k l}, \nabla_{y} w\right)_{Y}+\left(a\left(\nabla_{y} \kappa_{j k l}+e_{j} \theta_{k l}\right), e_{i} w\right)_{Y}\right. \\
& \left.+\left(a_{i j k l}^{2}-a_{i j}^{0} \theta_{k l}-a_{i j}^{0} b_{k l}^{0}, w\right)_{Y}\right\},
\end{aligned}
$$

for $Y$-periodic test functions $w \in \mathrm{H}_{\mathrm{per}}^{1}(Y)$. We now explain how the well-posedness of these cell problems leads to the definition of the effective tensors $a^{0}, a^{2}$ and $b^{0}$. To show that (2.12a) to $(2.12 \mathrm{~d})$ are well-posed in the quotient space $\mathcal{W}_{\text {per }}(Y)$, we apply Lax-Milgram theorem (we thus obtain a solution unique up to a constant). As the bilinear form $(v, w) \mapsto(a \nabla v, \nabla w)_{Y}$ is elliptic and bounded, we have to verify that the right hand sides belong to $\mathcal{W}_{\mathrm{per}}^{*}(Y)$. In other words, the right hand sides have to satisfy the solvability condition (1.8) and that gives constraints on the effective tensors. Let us now explicit these constraints. First, note that the right hand side of (2.12a) trivially satisfies this condition. Next, if we let $w=1$ in the right hand side of (2.12b), we obtain

$$
S_{i j}^{2}\left\{\left(a\left(\nabla \chi_{j}+e_{j}\right)-a^{0} e_{j}, e_{i}\right)_{Y}\right\}=|Y| S_{i j}^{2}\left\{\left\langle e_{i}^{T} a\left(\nabla \chi_{j}+e_{j}\right)\right\rangle_{Y}\right\}-|Y| S_{i j}^{2}\left\{a_{i j}^{0}\right\}=0,
$$

where we used the definition of the homogenized tensor (2.8). Hence, the cell problem (2.12b) is well-posed. Next, letting $w=1$ in the right hand side of (2.12c) we obtain

$$
S_{i j k}^{3}\left\{-\left(a \nabla \theta_{j k}, e_{i}\right)_{Y}-\left(e_{j} \chi_{k}, e_{i}\right)_{Y}+a_{i j}^{0}\left(\chi_{k}, 1\right)_{Y}\right\},
$$

and we need this quantity to vanish for any $1 \leq i, j, k \leq d$. Using the symmetry of $a$, equations (2.12a) with the test function $w=\theta_{j k}$ and $(2.12 \mathrm{~b})$ with $w=\chi_{i}$, we have

$$
\begin{aligned}
-\left(a \nabla \theta_{j k}, e_{i}\right)_{Y} & =\left(a \nabla \theta_{j k}, \nabla \chi_{i}\right)_{Y} \\
& =S_{j k}^{2}\left\{-\left(a e_{j} \chi_{k}, \nabla \chi_{i}\right)_{Y}+\left(a\left(\nabla \chi_{k}+e_{k}\right), e_{j} \chi_{i}\right)_{Y}-\left(a_{j k}^{0}, \chi_{i}\right)_{Y}\right\},
\end{aligned}
$$

and we can thus rewrite (2.14) as

$$
S_{i j k}^{3}\left\{-\left(a e_{j} \chi_{k}, \nabla \chi_{i}+e_{i}\right)_{Y}+\left(a\left(\nabla \chi_{k}+e_{k}\right), e_{j} \chi_{i}\right)_{Y}-a_{j k}^{0}\left(1, \chi_{i}\right)_{Y}+a_{i j}^{0}\left(\chi_{k}, 1\right)_{Y}\right\}=0 .
$$

It follows that the cell problem (2.12c) is well-posed. Finally, we apply the solvability condition (1.8) to the right hand side of equation $(2.12 \mathrm{~d})$ in order to obtain a constraint on $a^{2}$ and $b^{0}$. Letting $w=1$, we have

$$
|Y| S_{i j k l}^{4}\left\{a_{i j k l}^{2}-a_{i j}^{0} b_{k l}^{0}\right\}=S_{i j k l}^{4}\left\{-\left(a \nabla \kappa_{j k l}, e_{i}\right)_{Y}-\left(a e_{j} \theta_{k l}, e_{i}\right)_{Y}+\left(a_{i j}^{0}, \theta_{k l}\right)_{Y}\right\} .
$$

We use the symmetry of $a$, equation (2.12a) with test function $w=\kappa_{j k l}$ and equation (2.12c) with $w=\chi_{i}$ to get

$$
\begin{aligned}
-\left(a \nabla \kappa_{j k l}, e_{i}\right)_{Y} & =\left(a \nabla \kappa_{j k l}, \nabla \chi_{i}\right)_{Y} \\
& =S_{j k l}^{3}\left\{-\left(a e_{j} \theta_{k l}, \nabla \chi_{i}\right)_{Y}+\left(a\left(\nabla \theta_{k l}+e_{k} \chi_{l}\right), e_{j} \chi_{i}\right)_{Y}-a_{j k}^{0}\left(\chi_{l}, \chi_{i}\right)\right\},
\end{aligned}
$$

which combined with (2.15) gives (using the symmetry of $a$ )

$$
\begin{aligned}
|Y| S_{i j k l}^{4}\left\{a_{i j k l}^{2}-a_{i j}^{0} b_{k l}^{0}\right\}=S_{i j k l}^{4}\left\{\left(a e_{j} \chi_{i}, \nabla \theta_{k l}\right)_{Y}-\left(a\left(\nabla \chi_{i}+e_{i}\right), e_{j} \theta_{k l}\right)_{Y}+\left(a_{i j}^{0}, \theta_{k l}\right)_{Y}\right. \\
\left.-a_{j k}^{0}\left(\chi_{l}, \chi_{i}\right)_{Y}+\left(a e_{k} \chi_{l}, e_{j} \chi_{i}\right)_{Y}\right\} .
\end{aligned}
$$

Using equation $(2.12 \mathrm{~b})$ with test function $w=\theta_{k l}$, we obtain then the following constraint on $a^{2}$ and $b^{0}$

$$
|Y| S_{i j k l}^{4}\left\{a_{i j k l}^{2}-a_{i j}^{0} b_{k l}^{0}\right\}=S_{i j k l}^{4}\left\{\left(a_{j k} \chi_{l}, \chi_{i}\right)_{Y}-\left(a \nabla \theta_{j i}, \nabla \theta_{k l}\right)_{Y}-a_{j k}^{0}\left(\chi_{l}, \chi_{i}\right)_{Y}\right\} .
$$


We just showed that the cell problem $(2.12 \mathrm{~d})$ is well-posed in $\mathcal{W}_{\text {per }}(Y)$ if and only if the coefficients $a^{2}, b^{0}$ satisfy (2.16). In particular, if this constraint is satisfied, we can define the adaptation $\mathcal{B}^{\varepsilon} \tilde{u}$ as in (2.9) and show that $\left(\partial_{t}^{2}+\mathcal{A}^{\varepsilon}\right)\left(\mathcal{B}^{\varepsilon} \tilde{u}-u^{\varepsilon}\right)=\mathcal{O}\left(\varepsilon^{3}\right)$ (under sufficient regularity of $\tilde{u}$ and the correctors). Hence, applying Lemma 2.1 with $\mathcal{B}^{\varepsilon} \tilde{u}=\left[\mathcal{B}^{\varepsilon} \tilde{u}\right]$ leads to the estimate $\left\|u^{\varepsilon}-\tilde{u}\right\|_{L^{\infty}\left(0, T^{\varepsilon} ; L^{2}(\Omega)\right)} \leq C \varepsilon$ (as done in (2.6)). This result is rigorously proved in the next section.

Recall that we assumed $f=0$. It is in fact not necessary as we can also "correct" the terms coming from $f$ as follows. In order to cancel the non-vanishing terms $\varepsilon \chi_{i} \partial_{i} f+\varepsilon^{2}\left(b_{i j}^{0}+\theta_{i j}\right) \partial_{i j}^{2} f$ in (2.11), we add a correction term in the adaptation (2.9). Namely, we replace (2.9) by

$$
\begin{aligned}
\mathcal{B}^{\varepsilon}(\varphi ; \tilde{u})(t, x)= & \tilde{u}(t, x)+\varepsilon \chi_{i}\left(\frac{x}{\varepsilon}\right) \partial_{i} \tilde{u}(t, x)+\varepsilon^{2} \theta_{i j}\left(\frac{x}{\varepsilon}\right) \partial_{i j}^{2} \tilde{u}(t, x) \\
& +\varepsilon^{3} \kappa_{i j k}\left(\frac{x}{\varepsilon}\right) \partial_{i j k}^{3} \tilde{u}(t, x)+\varepsilon^{4} \kappa_{i j k l}\left(\frac{x}{\varepsilon}\right) \partial_{i j k l}^{4} \tilde{u}(t, x)+\varphi(t, x),
\end{aligned}
$$

where $\varphi(t, \cdot)$ belongs to the class $\varphi(t) \in \mathcal{W}_{\text {per }}(\Omega)$ that solves

$$
\begin{aligned}
& \left(\partial_{t}^{2}+\mathcal{A}^{\varepsilon}\right) \boldsymbol{\varphi}(t, x)=-\left[\varepsilon \chi_{i}\left(\frac{x}{\varepsilon}\right) \partial_{i} f(t, x)+\varepsilon^{2}\left(b_{i j}^{0}+\theta_{i j}\left(\frac{x}{\varepsilon}\right)\right) \partial_{i j}^{2} f(t, x)\right] \text { a.e. } t \in\left[0, T^{\varepsilon}\right], \\
& \boldsymbol{\varphi}(0, x)=\partial_{t} \boldsymbol{\varphi}(0, x)=[0] .
\end{aligned}
$$

The standard well-posedness of the wave equation ensures that if $f \in \mathrm{L}^{2}\left(0, T^{\varepsilon} ; \mathrm{H}^{2}(\Omega)\right)$ and $\chi_{i}, \theta_{i j} \in \mathcal{C}^{0}(\bar{Y})$, there exists a unique solution $\varphi$ of $(2.18)$, satisfying

$$
\boldsymbol{\varphi} \in \mathcal{C}\left(\left[0, T^{\varepsilon}\right] ; \mathcal{W}_{\text {per }}(\Omega)\right), \quad \partial_{t} \boldsymbol{\varphi} \in \mathcal{C}\left(\left[0, T^{\varepsilon}\right] ; \mathcal{L}^{2}(\Omega)\right), \quad \partial_{t}^{2} \boldsymbol{\varphi} \in \mathrm{L}^{2}\left(0, T^{\varepsilon} ; \mathcal{W}_{\text {per }}^{*}(\Omega)\right) .
$$

Observe then that $\mathcal{B}^{\varepsilon}(\varphi ; \tilde{u})$ defined in $(2.17)$ satisfies

$$
\left(\partial_{t}^{2}+\mathcal{A}^{\varepsilon}\right)\left(\left[\mathcal{B}^{\varepsilon}(\varphi ; \tilde{u})\right]-\left[u^{\varepsilon}\right]\right)=\left[r^{\varepsilon}(t)-\varepsilon \chi_{i}\left(\frac{x}{\varepsilon}\right) \partial_{i} f(t, x)-\varepsilon^{2}\left(b_{i j}^{0}+\theta_{i j}\left(\frac{x}{\varepsilon}\right)\right) \partial_{i j}^{2} f(t, x)\right],
$$

where $r^{\varepsilon}$ is the right hand side of $(2.11)$, so that $\left(\partial_{t}^{2}+\mathcal{A}^{\varepsilon}\right)\left(\left[\mathcal{B}^{\varepsilon}(\varphi ; \tilde{u})\right]-\left[u^{\varepsilon}\right]\right)=\mathcal{O}\left(\varepsilon^{3}\right)$. As we verify that $\partial_{t}\left[\mathcal{B}^{\varepsilon}(\varphi ; \tilde{u})\right](0)=\left[\mathcal{B}^{\varepsilon}\left(\varphi ; g^{1}\right)\right](0)$, the application of Lemma 2.1 leads to $\| \mathcal{B}^{\varepsilon} \tilde{u}-$ $\left[u^{\varepsilon}\right] \|_{\mathrm{L}^{\infty}\left(0, T^{\varepsilon} ; \mathcal{L}^{2}(\Omega)\right)}=\mathcal{O}(\varepsilon)$. However, in order to obtain an error estimate on $\left\|u^{\varepsilon}-\tilde{u}\right\|_{\mathrm{L}^{\infty}\left(\mathrm{L}^{2}\right)}$ (as done in (2.6)), we have to verify that the estimate $\left\|\left[\tilde{u}-\mathcal{B}^{\varepsilon}(\varphi ; \tilde{u})\right]\right\|_{\mathrm{L}^{\infty}\left(\mathcal{L}^{2}\right)} \leq C \varepsilon$ holds. Following the lines of Lemma 2.1, provided $\chi_{k} \in \mathcal{C}^{0}(\bar{Y}), \theta_{i j} \in \mathcal{C}^{0}(\bar{Y}), f \in \mathrm{L}^{2}\left(0, T^{\varepsilon} ; \mathrm{H}^{2}(\Omega)\right)$, we obtain the estimate

$$
\|\boldsymbol{\varphi}\|_{\mathrm{L}^{\infty}\left(0, T^{\varepsilon} ; \mathcal{L}^{2}(\Omega)\right)} \leq C \varepsilon\|f\|_{\mathrm{L}^{1}\left(0, T^{\varepsilon} ; \mathrm{H}^{2}(\Omega)\right)},
$$

where $C$ only depends on $\lambda, \Lambda, \max _{k}\left\|\chi_{k}\right\|_{\mathcal{C}^{0}(\bar{Y})}$ and $\max _{i j}\left\|\theta_{i j}\right\|_{\mathcal{C}^{0}(\bar{Y}) \text {. This estimate ensures }}$ that $\left\|\left[\tilde{u}-\mathcal{B}^{\varepsilon}(\varphi ; \tilde{u})\right]\right\|_{\mathrm{L}^{\infty}\left(\mathcal{L}^{2}\right)} \leq C \varepsilon\left(\|\tilde{u}\|_{\mathrm{L}^{\infty}\left(\mathrm{H}^{4}\right)}+\|f\|_{\mathrm{L}^{1}\left(0, T^{\varepsilon} ; \mathrm{H}^{2}(\Omega)\right)}\right)$.

To conclude this section, let us discuss the correctors and their dependence. First, as (2.12a$2.12 \mathrm{~d}$ ) are well-posed in $\mathcal{W}_{\text {per }}(Y)$, we obtain the unique (class of) solutions $\boldsymbol{\chi}_{k}, \boldsymbol{\theta}_{i j}, \boldsymbol{\kappa}_{i j k}, \boldsymbol{\rho}_{i j k l} \in$ $\mathcal{W}_{\text {per }}(Y)$ for $1 \leq i, j, k, l \leq d$. Note that $\boldsymbol{\theta}_{i j}$ depends on the choice $\chi_{k} \in \chi_{k}, \kappa_{i j k}$ depends on the choices $\chi_{k} \in \chi_{k}, \theta_{i j} \in \boldsymbol{\theta}_{i j}$, etc. A natural choice for the normalization of the correctors is the zero-mean function. However, observe that the constraint (2.16) has been derived independently of the choice of normalization. Hence, any normalization can be used.

\section{Main result : a priori error estimate and definition of the family of effective equations}

Let $a^{0} \in \operatorname{Sym}^{2}\left(\mathbb{R}^{d}\right)$ be the homogeneous tensor defined as (2.8) and let $b^{0} \in \mathrm{Ten}^{2}\left(\mathbb{R}^{d}\right)$ and $a^{2} \in \operatorname{Ten}^{4}\left(\mathbb{R}^{d}\right)$ be constant tensors such that

$$
\begin{array}{lll}
\text { i) } & b^{0} \in \operatorname{Sym}^{2}\left(\mathbb{R}^{d}\right), & b^{0} \eta \cdot \eta \geq 0 \quad \forall \eta \in \mathbb{R}^{d}, \\
\text { ii }) & a_{i j k l}^{2}=a_{l k j i}^{2}, & a^{2}\left(\eta \eta^{T}\right):\left(\eta \eta^{T}\right) \geq 0 \quad \forall \eta \in \mathbb{R}^{d} .
\end{array}
$$

Consider the following linear Boussinesq equation : we look for $\tilde{u}:\left[0, T^{\varepsilon}\right] \times \Omega \rightarrow \mathbb{R}$ such that

$$
\begin{array}{ll}
\partial_{t}^{2} \tilde{u}-a_{i j}^{0} \partial_{i j}^{2} \tilde{u}+\varepsilon^{2}\left(a_{i j k l}^{2} \partial_{i j k l}^{4} \tilde{u}-b_{i j}^{0} \partial_{i j}^{2} \partial_{t}^{2} \tilde{u}\right)=f & \text { in }\left(0, T^{\varepsilon}\right] \times \Omega \\
x \mapsto \tilde{u}(t, x) \Omega \text {-periodic } & \text { in }\left[0, T^{\varepsilon}\right], \\
\tilde{u}(0, x)=g^{0}(x), \quad \partial_{t} \tilde{u}(0, x)=g^{1}(x) & \text { in } \Omega,
\end{array}
$$


where the initial conditions $g^{0}, g^{1}$ and the source $f$ are the same as in the equation for $u^{\varepsilon}(1.9)$. As $a^{0}$ is symmetric and elliptic (see Ref. 7, 18) and under assumptions (3.1), the well-posedness of equation (3.2) can be proved as follows. Define the spaces

$$
\begin{aligned}
& \mathcal{H}=\left\{v \in \mathrm{L}_{0}^{2}(\Omega):\|v\|_{\mathrm{L}^{2}}^{2}+\varepsilon^{2}\left(b^{0} \nabla v, \nabla v\right)<\infty\right\}, \\
& \mathcal{V}=\left\{v \in \mathrm{W}_{\text {per }}(\Omega):\left(a^{0} \nabla v, \nabla v\right)_{\mathrm{L}^{2}}+\varepsilon^{2}\left(a^{2} \nabla^{2} v, \nabla^{2} v\right)<\infty\right\},
\end{aligned}
$$

and the bilinear forms

$$
\begin{aligned}
(v, w)_{\mathcal{H}} & =(v, w)_{\mathrm{L}^{2}(\Omega)}+\varepsilon^{2}\left(b^{0} \nabla v, \nabla w\right)_{\mathrm{L}^{2}(\Omega)}, \quad \forall v, w \in \mathcal{H}, \\
A(v, w) & =\left(a^{0} \nabla v, \nabla w\right)_{\mathrm{L}^{2}(\Omega)}+\varepsilon^{2}\left(a^{2} \nabla^{2} v, \nabla^{2} w\right)_{\mathrm{L}^{2}(\Omega)} \quad \forall v, w \in \mathcal{V},
\end{aligned}
$$

where $\nabla^{2} v$ denotes the Hessian matrix of $v$. We call a function $\tilde{u} \in \mathrm{L}^{\infty}\left(0, T^{\varepsilon} ; \mathcal{V}\right)$, with $\partial_{t} \tilde{u} \in$ $\mathrm{L}^{\infty}\left(0, T^{\varepsilon} ; \mathcal{H}\right)$, a weak solution of $(3.2)$ if for all test functions $v \in \mathcal{C}^{2}\left(\left[0, T^{\varepsilon}\right] ; \mathcal{V}\right)$, with $v\left(T^{\varepsilon}\right)=$ $\partial_{t} v\left(T^{\varepsilon}\right)=0, \tilde{u}$ satisfies

$$
\begin{aligned}
\int_{0}^{T^{\varepsilon}}\left(\tilde{u}(t), \partial_{t}^{2} v(t)\right)_{\mathcal{H}}+A(\tilde{u}(t), v(t)) \mathrm{d} t= & \int_{0}^{T^{\varepsilon}}(f(t), v(t))_{\mathrm{L}^{2}(\Omega)} \mathrm{d} t \\
& +\left(g^{1}, v(0)\right)_{\mathcal{H}}-\left(g^{0}, \partial_{t} v(0)\right)_{\mathcal{H}} .
\end{aligned}
$$

Following the Faedo-Galerkin method, we construct a sequence $\left\{u^{m}\right\}_{m \geq 0}$ and we show with an energy estimate that $\left\{u^{m}\right\}_{m \geq 0}$ is bounded in $\mathrm{L}^{\infty}\left(0, T^{\varepsilon} ; \mathcal{V}\right)$. We thus obtain the existence of a subsequence that weakly* converges in $\mathrm{L}^{\infty}\left(0, T^{\varepsilon} ; \mathcal{V}\right)$. We prove then that the weak* limit $\tilde{u}$ is the unique weak solution of (3.2). In order to derive the energy estimate, we need the assumptions (3.1). In particular, note that (3.1) ii) implies $\left(a^{2} \nabla^{2} v, \nabla^{2} v\right)_{L^{2}(\Omega)} \geq 0 v \in \mathcal{V}$.

The following theorem is our main result. It gives a sufficient condition on the coefficients $a^{2}, b^{0}$ such that $(3.2)$ is an effective equation up to times $\mathcal{O}\left(\varepsilon^{-2}\right)$.

Theorem 3.1. Assume that the $Y$-periodic tensor satisfies a $(y) \in \mathcal{C}^{2}(\bar{Y})$. Furthermore, assume that the solution $\tilde{u}$ of (3.2), the initial conditions and the right hand side satisfy the regularity

$$
\begin{gathered}
\tilde{u} \in \mathrm{L}^{\infty}\left(0, T^{\varepsilon} ; \mathrm{H}^{5}(\Omega)\right), \quad \partial_{t} \tilde{u} \in \mathrm{L}^{\infty}\left(0, T^{\varepsilon} ; \mathrm{H}^{4}(\Omega)\right), \quad \partial_{t}^{2} \tilde{u} \in \mathrm{L}^{\infty}\left(0, T^{\varepsilon} ; \mathrm{H}^{3}(\Omega)\right), \\
g^{0} \in \mathrm{H}^{4}(\Omega), \quad g^{1} \in \mathrm{H}^{3}(\Omega), \quad f \in \mathrm{L}^{2}\left(0, T^{\varepsilon} ; \mathrm{H}^{2}(\Omega)\right) .
\end{gathered}
$$

Let $\chi_{k}$ be the (class of) solution of $(2.12 \mathrm{a})$, fix any $\chi_{k} \in \chi_{k}$, let $\boldsymbol{\theta}_{i j}$ be the corresponding (class of) solution of $(2.12 \mathrm{~b})$ and $f i x \theta_{i j} \in \boldsymbol{\theta}_{i j}$. Assume then that $b^{0}$ and $a^{2}$ satisfy the relation

$$
S_{i j k l}^{4}\left\{a_{i j k l}^{2}-a_{i j}^{0} b_{k l}^{0}\right\}=S_{i j k l}^{4}\left\{\left\langle a_{j k} \chi_{l} \chi_{i}\right\rangle_{Y}-\left\langle a \nabla \theta_{j i} \cdot \nabla \theta_{k l}\right\rangle_{Y}-a_{j k}^{0}\left\langle\chi_{l} \chi_{i}\right\rangle_{Y}\right\} .
$$

Then, the following error estimate holds

$$
\begin{aligned}
\left\|u^{\varepsilon}-\tilde{u}\right\|_{\mathrm{L}^{\infty}\left(0, T^{\varepsilon} ; \mathrm{L}^{2}(\Omega)\right)} \leq C \varepsilon & \left\|g^{1}\right\|_{\mathrm{H}^{3}(\Omega)}+\left\|g^{0}\right\|_{\mathrm{H}^{4}(\Omega)}+\|f\|_{\mathrm{L}^{1}\left(0, T^{\varepsilon} ; \mathrm{H}^{2}(\Omega)\right)} \\
& \left.+\|\tilde{u}\|_{\mathrm{L}^{\infty}\left(0, T^{\varepsilon} ; \mathrm{H}^{5}(\Omega)\right)}+\left\|\partial_{t}^{2} \tilde{u}\right\|_{\mathrm{L}^{\infty}\left(0, T^{\varepsilon} ; \mathrm{H}^{3}(\Omega)\right)}\right),
\end{aligned}
$$

where $C$ depends only on $T, Y, a, \lambda$ and $\Lambda$.

Let us emphasize that the constant $C$ in estimate (3.5) does not depend on $\Omega$. Hence, for an arbitrarily large domain $\Omega$, if the quantities

$$
\left\|g^{1}\right\|_{\mathrm{H}^{3}(\Omega)},\left\|g^{0}\right\|_{\mathrm{H}^{4}(\Omega)},\|f\|_{\mathrm{L}^{1}\left(0, T^{\varepsilon} ; \mathrm{H}^{2}(\Omega)\right)},\|\tilde{u}\|_{\mathrm{L}^{\infty}\left(0, T^{\varepsilon} ; \mathrm{H}^{5}(\Omega)\right)},\left\|\partial_{t}^{2} \tilde{u}\right\|_{\mathrm{L}^{\infty}\left(0, T^{\varepsilon} ; \mathrm{H}^{3}(\Omega)\right)},
$$

are bounded independently of $\varepsilon$, estimate $(3.5)$ reads $\left\|u^{\varepsilon}-\tilde{u}\right\|_{\mathrm{L}^{\infty}\left(0, T^{\varepsilon} ; \mathrm{L}^{2}(\Omega)\right)}=\mathcal{O}(\varepsilon)$.

Thanks to Theorem 3.1, we can define the family of effective equations.

Definition 3.1. The family $\mathcal{E}$ of effective equations is the set of equations $(3.2)$ where $b^{0}, a^{2}$ satisfy both (3.1) and (3.4). Note that $\mathcal{E}$ is used to denote both the family of effective equations and the corresponding solutions.

The proof of Theorem 3.1 follows two steps. We first define the adaptation operator $\mathcal{B}^{\varepsilon}$ using the correctors defined in Section 2.2. Then, we show that $\mathcal{B}^{\varepsilon} \tilde{u}$ satisfies the same wave 
equation as $u^{\varepsilon}$ up to a remainder of order $\mathcal{O}\left(\varepsilon^{3}\right)$ (Lemma 3.1). Finally, we use the triangle inequality and apply Lemma 2.1 to obtain the error estimate.

First, note that the cell problems (2.12a) and (2.12b) are well-posed ( $a^{0}$ is defined as (2.8)). Then, as (3.4) is equivalent to (2.16), the cell problems (2.12c) and (2.12d) are well-posed. Let $\chi_{i}$ and $\theta_{i j}$ be as in Theorem 3.1, let $\boldsymbol{\kappa}_{i j k}$ be the corresponding solution of (2.12c), fix $\kappa_{i j k} \in \boldsymbol{\kappa}_{i j k}$, and similarly fix $\rho_{i j k l}$ in the corresponding class $\boldsymbol{\rho}_{i j k l}$ of solution of (2.12d). As we assume $a \in \mathcal{C}^{2}(\bar{Y})$ (in Theorem 3.1), elliptic regularity result (see Ref. 8) and Sobolev embeddings ensure that $\chi_{i}, \theta_{i j}, \kappa_{i j k}, \rho_{i j k l} \in \mathcal{C}^{1}(\bar{Y})$ and for any $1 \leq i, j, k, l \leq d$ it holds

$$
\left\|\chi_{i}\right\|_{\mathcal{C}^{1}(\bar{Y})},\left\|\theta_{i j}\right\|_{\mathcal{C}^{1}(\bar{Y})},\left\|\kappa_{i j k}\right\|_{\mathcal{C}^{1}(\bar{Y})},\left\|\rho_{i j k l}\right\|_{\mathcal{C}^{1}(\bar{Y})} \leq C \max _{i j}\left\|a_{i j}\right\|_{\mathcal{C}^{2}(\bar{Y})},
$$

where $C$ depends only on $\lambda, \Lambda$ in (1.10) and $Y$. Finally, let $\varphi \in \mathcal{C}^{0}\left(\left[0, T^{\varepsilon}\right] ; \mathcal{W}_{\text {per }}(\Omega)\right)$ be the unique (class of) solution of (2.18).

We now define the adaptation operator as

$$
\begin{aligned}
\mathcal{B}^{\varepsilon}(\boldsymbol{\varphi} ; \cdot): & \mathrm{L}^{2}\left(0, T^{\varepsilon} ; \mathrm{H}_{\mathrm{per}}^{1}(\Omega) \cap \mathrm{H}^{3}(\Omega)\right) \rightarrow \mathrm{L}^{2}\left(0, T^{\varepsilon} ; \mathcal{W}_{\mathrm{per}}^{*}(\Omega)\right), \quad v \mapsto \mathcal{B}^{\varepsilon}(\boldsymbol{\varphi} ; v), \\
\left\langle\mathcal{B}^{\varepsilon}(\boldsymbol{\varphi} ; v)\right. & (t), \boldsymbol{w}\rangle_{\mathcal{W}_{\mathrm{per}}^{*}, \mathcal{W}_{\mathrm{per}}}= \\
& \left(\left[v(t)+\varepsilon\left(\chi_{j}-\partial_{y_{m}} \theta_{m j}\right) \partial_{j} v(t)+\varepsilon^{3}\left(\kappa_{j k l}-\partial_{y_{m}} \rho_{m j k l}\right) \partial_{j k l}^{3} v(t)\right], \boldsymbol{w}\right)_{\mathcal{L}^{2}(\Omega)} \\
& -\left(\varepsilon^{2} \theta_{m j} \partial_{j} v(t)+\varepsilon^{4} \rho_{m j k l} \partial_{j k l}^{3} v(t), \partial_{m} \boldsymbol{w}\right)_{\mathrm{L}^{2}(\Omega)}+\langle\boldsymbol{\varphi}(t), \boldsymbol{w}\rangle_{\mathcal{W}_{\mathrm{per}}^{*}, \mathcal{W}_{\mathrm{per}}}
\end{aligned}
$$

for a.e. $t \in\left[0, T^{\varepsilon}\right]$, where the correctors $\chi_{i}, \theta_{i j}, \kappa_{i j k}$ and $\rho_{i j k l}$ are evaluated at $y=\frac{x}{\varepsilon}$. Using the Green formula (as in Remark 3.1), we verify that for $v \in \mathrm{L}^{2}\left(0, T^{\varepsilon} ; \mathrm{H}_{\text {per }}^{1}(\Omega) \cap \mathrm{H}^{4}(\Omega)\right.$ ), we have $\left\langle\mathcal{B}^{\varepsilon}(\boldsymbol{\varphi} ; v)(t), \boldsymbol{w}\right\rangle=\left(\left[\left(\mathcal{B}^{\varepsilon}(\varphi ; v)(t)\right], \boldsymbol{w}\right) \mathcal{\mathcal { S }}^{2}\right.$ where $\mathcal{B}^{\varepsilon}(\varphi ; \cdot)$ is defined in $(2.17)$. Furthermore, note that for $v \in \mathrm{H}^{2}\left(0, T^{\varepsilon} ; \mathrm{H}_{\mathrm{per}}^{1}(\Omega) \cap \mathrm{H}^{3}(\Omega)\right)$ it holds $\partial_{t}^{2} \mathcal{B}^{\varepsilon}(\boldsymbol{\varphi} ; v)=\mathcal{B}^{\varepsilon}\left(\partial_{t}^{2} \boldsymbol{\varphi} ; \partial_{t}^{2} v\right)$. Finally, note that under the assumptions of Theorem 3.1, $\mathcal{B}^{\varepsilon}(\boldsymbol{\varphi} ; \cdot)$ verifies the hypotheses of Lemma 2.1.

Remark 3.1. The following formula (applications of the Green formula) will be useful : for any $Y$-periodic $c \in\left[\mathcal{C}^{1}(\bar{Y})\right]^{d}, v \in \mathrm{H}_{\mathrm{per}}^{1}(\Omega)$ and $\boldsymbol{w}=[w] \in \mathcal{W}_{\text {per }}(\Omega)$,

$$
\begin{aligned}
& \left(\left[\varepsilon c_{k}(\dot{\bar{\varepsilon}}) \partial_{k} v\right], \boldsymbol{w}\right)_{\mathcal{L}^{2}(\Omega)}=\left(\varepsilon c_{k}(\dot{\bar{\varepsilon}}) \partial_{k} v, w\right)_{\mathrm{L}^{2}(\Omega)}-|\Omega|\left\langle\varepsilon c_{k}(\dot{\bar{\varepsilon}}) \partial_{k} v\right\rangle_{\Omega}\langle w\rangle_{\Omega} \\
& \quad=-\left(\partial_{y_{m}} c_{m}(\dot{\bar{\varepsilon}}) v, w\right)_{\mathrm{L}^{2}(\Omega)}-\left(\varepsilon c_{m}(\dot{\dot{\varepsilon}}) v, \partial_{m} w\right)_{\mathrm{L}^{2}(\Omega)}+|\Omega|\left\langle\partial_{y_{m}} c_{m}(\dot{\bar{\varepsilon}}) v\right\rangle_{\Omega}\langle w\rangle_{\Omega} \\
& \quad=-\left(\left[\partial_{y_{m}} c_{m}(\dot{\bar{\varepsilon}}) v\right], \boldsymbol{w}\right)_{\mathcal{L}^{2}(\Omega)}-\left(\varepsilon c_{m}(\dot{\bar{\varepsilon}}) v, \partial_{m} \boldsymbol{w}\right)_{\mathrm{L}^{2}(\Omega)},
\end{aligned}
$$

where we recall the notation $\partial_{y_{m}} c_{m}=\sum_{m=1}^{d} \partial_{y_{m}} c_{m}$.

Lemma 3.1. Under the assumptions of Theorem 3.1, $\mathcal{B}^{\varepsilon}(\varphi ; \tilde{u})$ satisfies

$$
\left(\partial_{t}^{2}+\mathcal{A}^{\varepsilon}\right) \mathcal{B}^{\varepsilon}(\boldsymbol{\varphi} ; \tilde{u})(t)=[f(t)]+\mathcal{R}^{\varepsilon} \tilde{u}(t) \quad \text { in } \mathcal{W}_{\mathrm{per}}^{*}(\Omega) \quad \text { for a.e. } t \in\left[0, T^{\varepsilon}\right],
$$

where the remainder $\mathcal{R}^{\varepsilon} \tilde{u} \in \mathrm{L}^{\infty}\left(0, T^{\varepsilon} ; \mathcal{W}_{\text {per }}^{*}(\Omega)\right)$ satisfies the estimate

$$
\left\|\boldsymbol{\mathcal { R }}^{\varepsilon} \tilde{u}\right\|_{\mathrm{L}^{\infty}\left(0, T^{\varepsilon} ; \mathcal{W}_{\mathrm{per}}^{*}(\Omega)\right)} \leq C \varepsilon^{3}\left(\|\tilde{u}\|_{\mathrm{L}^{\infty}\left(0, T^{\varepsilon} ; \mathrm{H}^{5}(\Omega)\right)}+\left\|\partial_{t}^{2} \tilde{u}\right\|_{\mathrm{L}^{\infty}\left(0, T^{\varepsilon} ; \mathrm{H}^{3}(\Omega)\right)}\right),
$$

for a constant $C$ that only depends on $\lambda, \Lambda$, a and $Y$.

Proof. To simplify the notation, $\langle\cdot, \cdot\rangle_{\mathcal{W}_{\text {per }}^{*}}, \mathcal{W}_{\text {per }}$ is denoted by $\langle\cdot, \cdot\rangle$. First, using equation $(3.2)$ and the assumptions on the regularity of $\tilde{u}$, note that the following equalities hold in $\mathrm{L}^{2}(\Omega)$ for a.e. $t \in\left[0, T^{\varepsilon}\right]$ and for $1 \leq p \leq d$,

$$
\begin{aligned}
& \partial_{t}^{2} \tilde{u}=f+a_{i j}^{0} \partial_{i j}^{2} \tilde{u}-\varepsilon^{2} a_{i j k l}^{2} \partial_{i j k l}^{4} \tilde{u}+\varepsilon^{2} b_{i j}^{0} \partial_{i j}^{2} \partial_{t}^{2} \tilde{u}, \\
& \partial_{p} \partial_{t}^{2} \tilde{u}=\partial_{p} f+a_{i j}^{0} \partial_{p i j}^{3} \tilde{u}-\varepsilon^{2} a_{i j k l}^{2} \partial_{p i j k l}^{5} \tilde{u}+\varepsilon^{2} b_{i j}^{0} \partial_{p i j}^{3} \partial_{t}^{2} \tilde{u} .
\end{aligned}
$$

Then, we fix $t \in\left[0, T^{\varepsilon}\right]$ and develop the terms $\partial_{t}^{2} \mathcal{B}^{\varepsilon}(\boldsymbol{\varphi} ; \tilde{u})(t)$ and $\mathcal{A}^{\varepsilon} \mathcal{B}^{\varepsilon}(\boldsymbol{\varphi} ; \tilde{u})(t)$ separately. Using (3.10) and formula (3.8), we have

$$
\left(\left[\partial_{t}^{2} \tilde{u}\right], \boldsymbol{w}\right)_{\mathcal{L}^{2}}=\left(\left[f+a_{i j}^{0} \partial_{i j}^{2} \tilde{u}-\varepsilon^{2} a_{i j k l}^{2} \partial_{i j k l}^{4} \tilde{u}\right], \boldsymbol{w}\right)_{\mathcal{L}^{2}}-\left(\varepsilon^{2} b_{m j}^{0} \partial_{j} \partial_{t}^{2} \tilde{u}, \partial_{m} \boldsymbol{w}\right)_{L^{2}} .
$$


As $\partial_{t}^{2} \mathcal{B}^{\varepsilon}(\boldsymbol{\varphi} ; \tilde{u})(t)=\mathcal{B}^{\varepsilon}\left(\partial_{t}^{2} \boldsymbol{\varphi} ; \partial_{t}^{2} \tilde{u}\right)(t)$ in $\mathcal{W}_{\text {per }}^{*}(\Omega)$, using (3.7) and (3.12) we obtain

$$
\begin{aligned}
\left\langle\partial_{t}^{2} \mathcal{B}^{\varepsilon}(\boldsymbol{\varphi} ; \tilde{u}), \boldsymbol{w}\right\rangle= & \left(\left[f+a_{i j}^{0} \partial_{i j}^{2} \tilde{u}+\varepsilon\left(\chi_{j}-\partial_{y_{m}} \theta_{m j}\right) \partial_{j} \partial_{t}^{2} \tilde{u}-\varepsilon^{2} a_{i j k l}^{2} \partial_{i j k l}^{4} \tilde{u}\right.\right. \\
& \left.\left.+\varepsilon^{3}\left(\kappa_{j k l}-\partial_{y_{m}} \rho_{m j k l}\right) \partial_{j k l}^{3} \partial_{t}^{2} \tilde{u}\right], \boldsymbol{w}\right)_{\mathcal{L}^{2}} \\
& -\left(\varepsilon^{2}\left(\theta_{m j}+b_{m j}^{0}\right) \partial_{j} \partial_{t}^{2} \tilde{u}+\varepsilon^{4} \rho_{m j k l} \partial_{j k l}^{3} \partial_{t}^{2} \tilde{u}, \partial_{m} \boldsymbol{w}\right)_{\mathrm{L}^{2}}+\left\langle\partial_{t}^{2} \boldsymbol{\varphi}, \boldsymbol{w}\right\rangle .
\end{aligned}
$$

Using now (3.11) to substitute $\partial_{j} \partial_{t}^{2} \tilde{u}$, we obtain

$$
\begin{aligned}
\left\langle\partial_{t}^{2} \mathcal{B}^{\varepsilon}(\boldsymbol{\varphi} ; \tilde{u}), \boldsymbol{w}\right\rangle= & \left(\left[f+a_{i j}^{0} \partial_{i j}^{2} \tilde{u}+\varepsilon a_{i j}^{0}\left(\chi_{k}-\partial_{y_{m}} \theta_{m k}\right) \partial_{i j k}^{3} \partial_{t}^{2} \tilde{u}-\varepsilon^{2} a_{i j k l}^{2} \partial_{i j k l}^{4} \tilde{u}\right], \boldsymbol{w}\right)_{\mathcal{L}^{2}} \\
& -\left(\varepsilon^{2} a_{i j}^{0}\left(\theta_{m k}+b_{m k}^{0}\right) \partial_{i j k}^{3} \tilde{u}, \partial_{m} \boldsymbol{w}\right)_{\mathrm{L}^{2}} \\
& +\left(\left[\varepsilon\left(\chi_{k}-\partial_{y_{m}} \theta_{m k}\right) \partial_{k} f\right], \boldsymbol{w}\right)_{\mathcal{L}^{2}}-\left(\varepsilon^{2}\left(\theta_{m j}+b_{m j}^{0}\right) \partial_{j} f, \partial_{m} \boldsymbol{w}\right)_{\mathrm{L}^{2}} \\
& +\left\langle\partial_{t}^{2} \boldsymbol{\varphi}, \boldsymbol{w}\right\rangle+\left\langle\boldsymbol{\mathcal { R }}_{1}^{\varepsilon} \tilde{u}, \boldsymbol{w}\right\rangle,
\end{aligned}
$$

where

$$
\begin{aligned}
\left\langle\boldsymbol{\mathcal { R }}_{1}^{\varepsilon} \tilde{u}, \boldsymbol{w}\right\rangle= & \left(\left[\varepsilon^{3}\left(\kappa_{j k l}+b_{j k}^{0} \chi_{l}-\partial_{y_{m}}\left(\rho_{m j k l}+b_{j k}^{0} \theta_{m l}\right)\right) \partial_{j k l}^{3} \partial_{t}^{2} \tilde{u}\right.\right. \\
& \left.\left.-\varepsilon^{3} a_{i j k l}^{2}\left(\chi_{p}-\partial_{y_{m}} \theta_{m p}\right) \partial_{i j k l p}^{5} \tilde{u}\right], \boldsymbol{w}\right)_{\mathcal{L}^{2}} \\
& +\left(\varepsilon^{4}\left(\rho_{m i j k}-b_{i j}^{0} \theta_{m k}+b_{i j}^{0} b_{m k}^{0}\right) \partial_{i j k}^{3} \partial_{t}^{2} \tilde{u}+\varepsilon^{4} a_{i j k l}^{2}\left(\theta_{m p}+b_{m p}^{0}\right) \partial_{i j k l p}^{5} \tilde{u}, \partial_{m} \boldsymbol{w}\right)_{\mathcal{L}^{2}} .
\end{aligned}
$$

Finally, applying formula (3.8), we obtain

$$
\begin{aligned}
\left\langle\partial_{t}^{2} \mathcal{B}^{\varepsilon}(\boldsymbol{\varphi} ; \tilde{u}), \boldsymbol{w}\right\rangle= & \left(\left[f+a_{i j}^{0} \partial_{i j}^{2} \tilde{u}+\varepsilon a_{i j}^{0} \chi_{k} \partial_{i j k}^{3} \tilde{u}+\varepsilon^{2}\left(a_{i j}^{0} \theta_{k l}+a_{i j}^{0} b_{k l}^{0}-a_{i j k l}^{2}\right) \partial_{i j k l}^{4} \tilde{u}\right], \boldsymbol{w}\right)_{\mathcal{L}^{2}} \\
& +\left(\left[\varepsilon \chi_{k} \partial_{k} f+\varepsilon^{2}\left(\theta_{i j}+b_{i j}^{0}\right) \partial_{i j}^{2} f\right], \boldsymbol{w}\right)_{\mathcal{L}^{2}}+\left\langle\partial_{t}^{2} \boldsymbol{\varphi}, \boldsymbol{w}\right\rangle+\left\langle\boldsymbol{\mathcal { R }}_{1}^{\varepsilon} \tilde{u}, \boldsymbol{w}\right\rangle .
\end{aligned}
$$

Next, the second term is computed as

$$
\begin{aligned}
& \left\langle\mathcal{A}^{\varepsilon} \mathcal{B}^{\varepsilon}(\boldsymbol{\varphi} ; \tilde{u}), \boldsymbol{w}\right\rangle=\left(\left[\quad \varepsilon^{-1}\left(-\nabla_{y} \cdot\left(a\left(\nabla_{y} \chi_{k}+e_{k}\right)\right)\right) \partial_{k} \tilde{u}\right.\right. \\
& +\quad\left(-\nabla_{y} \cdot\left(a\left(\nabla_{y} \theta_{i j}+e_{i} \chi_{j}\right)\right)-e_{i}^{T} a\left(\nabla_{y} \chi_{j}+e_{j}\right)\right) \partial_{i j}^{2} \tilde{u} \\
& +\varepsilon^{1}\left(-\nabla_{y} \cdot\left(a\left(\nabla_{y} \kappa_{i j k}+e_{i} \theta_{j k}\right)\right)-e_{i}^{T} a\left(\nabla_{y} \theta_{j k}+e_{j} \chi_{k}\right)\right) \partial_{i j k}^{3} \tilde{u} \\
& \left.\left.+\varepsilon^{2}\left(-\nabla_{y} \cdot\left(a\left(\nabla_{y} \rho_{i j k l}+e_{i} \kappa_{j k l}\right)\right)-e_{i}^{T} a\left(\nabla_{y} \kappa_{j k l}+e_{j} \theta_{k l}\right)\right) \partial_{i j k l}^{4} \tilde{u}\right], \boldsymbol{w}\right)_{\mathcal{L}^{2}} \\
& +\left\langle\mathcal{A}^{\varepsilon} \boldsymbol{\varphi}, \boldsymbol{w}\right\rangle+\left\langle\boldsymbol{\mathcal { R }}_{2}^{\varepsilon} \tilde{u}, \boldsymbol{w}\right\rangle,
\end{aligned}
$$

where

$$
\left\langle\boldsymbol{\mathcal { R }}_{2}^{\varepsilon} \tilde{u}, \boldsymbol{w}\right\rangle=\varepsilon^{3}\left(\left[-e_{i}^{T} a\left(\nabla_{y} \rho_{j k l p}+e_{j} \kappa_{k l p}\right) \partial_{i j k l p}^{5} \tilde{u}\right], \boldsymbol{w}\right)_{\mathcal{L}^{2}}+\left(a_{m i} \rho_{j k l p} \partial_{i j k l p}^{5} \tilde{u}, \partial_{m} \boldsymbol{w}\right)_{\mathrm{L}^{2}} .
$$

Now, we combine (3.13) and (3.14) and use cell problems (2.12a-2.12d) and (2.18) and obtain $\left(\partial_{t}^{2}+\mathcal{A}^{\varepsilon}\right) \mathcal{B}^{\varepsilon}(\boldsymbol{\varphi} ; \tilde{u})(t)=[f(t)]+\boldsymbol{\mathcal { R }}^{\varepsilon} \tilde{u}(t)$, where $\boldsymbol{\mathcal { R }}^{\varepsilon} \tilde{u}=\boldsymbol{\mathcal { R }}_{1}^{\varepsilon} \tilde{u}+\boldsymbol{\mathcal { R }}_{2}^{\varepsilon} \tilde{u}$. Thanks to the regularity of the correctors and using (3.6), we verify estimate (3.9) for the remainder $\boldsymbol{\mathcal { R }}^{\varepsilon} \tilde{u}$ and the proof of the lemma is complete.

Proof of Theorem 3.1. As $\langle\tilde{u}\rangle_{\Omega}=\left\langle u^{\varepsilon}\right\rangle_{\Omega}$ it holds $\left\|u^{\varepsilon}-\tilde{u}\right\|_{L^{\infty}\left(L^{2}\right)}=\left\|\left[u^{\varepsilon}-\tilde{u}\right]\right\|_{L^{\infty}\left(\mathcal{L}^{2}\right)}$, hence using the triangle inequality we split the error as

$$
\left\|u^{\varepsilon}-\tilde{u}\right\|_{L^{\infty}\left(L^{2}\right)} \leq\left\|\mathcal{B}^{\varepsilon} \tilde{u}-\left[u^{\varepsilon}\right]\right\|_{L^{\infty}\left(\mathcal{L}^{2}\right)}+\left\|[\tilde{u}]-\mathcal{B}^{\varepsilon} \tilde{u}\right\|_{L^{\infty}\left(\mathcal{L}^{2}\right)} .
$$

Let us bound the two terms of the right hand side. The equation for $u^{\varepsilon}(1.9)$ implies that $\left(\partial_{t}^{2}+\mathcal{A}^{\varepsilon}\right)\left[u^{\varepsilon}(t)\right]=[f(t)]$ in $\mathcal{W}_{\text {per }}^{*}(\Omega)$ for a.e. $t \in\left[0, T^{\varepsilon}\right]$. Lemma 3.1 implies thus that

$$
\left(\partial_{t}^{2}+\mathcal{A}^{\varepsilon}\right)\left(\mathcal{B}^{\varepsilon}(\boldsymbol{\varphi} ; \tilde{u})-\left[u^{\varepsilon}\right]\right)(t)=\boldsymbol{\mathcal { R }}^{\varepsilon} \tilde{u}(t) \text { in } \mathcal{W}_{\text {per }}^{*}(\Omega) \text { for a.e. } t \in\left[0, T^{\varepsilon}\right] .
$$


Applying Lemma 2.1, using estimate (3.9) and the definition of $\mathcal{B}^{\varepsilon}(\boldsymbol{\varphi} ; \cdot)$ in $(3.7)$, we obtain

$$
\left\|\mathcal{B}^{\varepsilon}(\boldsymbol{\varphi} ; \tilde{u})-\left[u^{\varepsilon}\right]\right\|_{\mathrm{L}^{\infty}\left(\mathcal{L}^{2}\right)} \leq C \varepsilon\left(\left\|g^{1}\right\|_{\mathrm{H}^{3}}+\left\|g^{0}\right\|_{\mathrm{H}^{4}}+\|\tilde{u}\|_{\mathrm{L}^{\infty}\left(\mathrm{H}^{5}\right)}+\left\|\partial_{t}^{2} \tilde{u}\right\|_{\mathrm{L}^{\infty}\left(\mathrm{H}^{3}\right)}\right)
$$

where $C$ depends only on $\lambda, \Lambda, a, Y$ and $T$. For the second term of (3.15), we use the definition of $\mathcal{B}^{\varepsilon}(\boldsymbol{\varphi} ; \cdot)(3.7)$ and estimate $(2.20)$ and obtain

$$
\left\|[\tilde{u}]-\mathcal{B}^{\varepsilon}(\boldsymbol{\varphi} ; \tilde{u})\right\|_{\mathrm{L}^{\infty}\left(\mathcal{L}^{2}\right)} \leq C \varepsilon\left(\|\tilde{u}\|_{\mathrm{L}^{\infty}\left(\mathrm{H}^{4}\right)}+\|f\|_{\mathrm{L}^{1}\left(\mathrm{H}^{2}\right)}\right),
$$

where $C$ depends only on $\lambda, \Lambda, a$ and $Y$. Combining (3.15), (3.16) and (3.17), we obtain estimate (3.5) and the proof of Theorem 3.1 is complete.

\section{Computing the tensors of an effective equation}

In Definition 3.1 a family of effective solutions $\mathcal{E}$ is defined in an implicit way. This does not yet give a way to compute $a^{2}$ and $b^{0}$, nor even ensure the existence of an effective equation. In this section, we prove in a constructive way that there exists coefficients $a^{2}, b^{0}$ satisfying both (3.4) and (3.1). In the one-dimensional case, we show that Theorem 3.1 reduces to the result obtained in Ref. 4, where a family of such functions is defined in an explicit way. In the multidimensional case, we will give an algorithm to compute the coefficients to obtain an effective solution (the algorithm can be easily modified to obtain other effective solutions).

\section{One-dimensional case}

The computation of the effective coefficients in the one-dimensional case is very particular. As showed in Ref. 4, the coefficients $b^{0}$ and $a^{2}$ in the effective equation (3.2) can be computed with the solution of one single cell problem. That leads to the explicit parametric definition of a family of effective equations. For completeness, we show here how this result is obtained with Theorem 3.1 .

Let us rewrite the constraint (3.4) on the coefficients $b^{0}, a^{2}$ as

$$
|Y|\left(a^{2}-a^{0} b^{0}\right)=\left(a(y)\left(\partial_{y} \theta+\chi\right), \chi-\partial_{y} \theta\right)_{Y}-a^{0}(\chi, \chi)_{Y} .
$$

We now derive two relations that are only valid in the one-dimensional case. Noting that $a\left(\partial_{y} \chi+1\right) \in \mathrm{H}(\operatorname{div}, Y)$, we use integration by parts, the periodicity of $a\left(\partial_{y} \chi+1\right)$ and the cell problem for $\chi(2.12 \mathrm{a})$ to obtain for any $y_{1}, y_{2} \in Y$

$$
\left.a\left(\partial_{y} \chi+1\right)\right|_{y_{1}} ^{y_{2}}=\left.a\left(\partial_{y} \chi+1\right)\left(H_{y_{2}}-H_{y_{1}}\right)\right|_{\partial Y}-\int_{Y}\left(H_{y_{2}}-H_{y_{1}}\right) \partial_{y}\left(a\left(\partial_{y} \chi+1\right)\right) \mathrm{d} y=0,
$$

where $H_{y_{i}}$ is the Heaviside step function centered in $y_{i}$. Hence, $a\left(\partial_{y} \chi+1\right)$ is constant on $Y$ and thanks to the definition of $a^{0}$ we conclude that $a(y)\left(\partial_{y} \chi(y)+1\right)=a^{0} \forall y \in Y$. In a similar way, using this equality in the cell problem for $\theta(2.12 \mathrm{~b})$, we verify that $a(y)\left(\partial_{y} \theta(y)+\chi(y)\right)=C$ is constant on $Y$. Dividing this equality by $a(y)$, taking the mean over $Y$ and using that $\langle 1 / a\rangle_{Y}=1 / a^{0}$, we verify that $C=a^{0}\langle\chi\rangle_{Y}$. This equality used in (4.1) leads to a constraint independent of $\theta$ :

$$
a^{2}-a^{0} b^{0}=a^{0}\langle\chi\rangle_{Y}\left\langle\chi-\partial_{y} \theta\right\rangle_{Y}-a^{0}\left\langle\chi^{2}\right\rangle_{Y}=a^{0}\langle\chi\rangle_{Y}^{2}-a^{0}\left\langle\chi^{2}\right\rangle_{Y}
$$

Now observe that any non-negative $b^{0}, a^{2}$ satisfying (4.2) can be written as

$$
b^{0}=\left\langle\left(\chi-\langle\chi\rangle_{Y}\right)^{2}\right\rangle_{Y}+\langle\chi\rangle_{Y}^{2}, \quad a^{2}=a^{0}\langle\chi\rangle_{Y}^{2},
$$

where we note that $\left\langle\left(\chi-\langle\chi\rangle_{Y}\right)^{2}\right\rangle$ is independent of $\langle\chi\rangle_{Y}$. We can then explicitly define parametrically the family of effective solutions as

$$
\mathcal{E}=\left\{\tilde{u}_{\langle\chi\rangle} \text { solution of }(3.2) \text { where } b^{0}, a^{2} \text { are defined as in }(4.3)\right\}
$$

Observe that for $\langle\chi\rangle_{Y}=0$, the coefficient $a^{2}$ vanishes and hence there is no fourth order operator $a^{2} \partial_{x}^{4}$ in the effective equation. This particular equation was the one given in Ref. 19. It is also the effective model on which the FE-HMM-L from Ref. 4 is based. 


\section{Multidimensional case}

In order to obtain an effective equation, following Definition 3.1, we look for a pair of tensors $b^{0} \in \operatorname{Ten}^{2}\left(\mathbb{R}^{d}\right), a^{2} \in \operatorname{Ten}^{4}\left(\mathbb{R}^{d}\right)$ such that

$$
\begin{aligned}
& b^{0} \in \operatorname{Sym}^{2}\left(\mathbb{R}^{d}\right), \quad b^{0} \eta \cdot \eta \geq 0 \quad \forall \eta \in \mathbb{R}^{d}, \\
& a_{i j k l}^{2}=a_{l k j i}^{2}, \quad a^{2}\left(\eta \eta^{T}\right):\left(\eta \eta^{T}\right) \geq 0 \quad \forall \eta \in \mathbb{R}^{d}, \\
& S_{i j k l}^{4}\left\{a_{i j k l}^{2}-a_{i j}^{0} b_{k l}^{0}\right\}=S_{i j k l}^{4}\left\{\left\langle a_{j k} \chi_{l} \chi_{i}\right\rangle_{Y}-\left\langle a \nabla \theta_{j i} \cdot \nabla \theta_{k l}\right\rangle_{Y}-a_{j k}^{0}\left\langle\chi_{l} \chi_{i}\right\rangle_{Y}\right\},
\end{aligned}
$$

where $\chi_{i} \in \chi_{i}, \theta_{i j} \in \boldsymbol{\theta}_{i j}$ and $\boldsymbol{\chi}_{i}, \boldsymbol{\theta}_{i j}$ are the unique (class of) solutions of the cell problems (2.12a) and (2.12b), respectively. In the multidimensional case, constructing a pair $a^{2}, b^{0}$ satisfying (4.4) is not as straightforward as in the one-dimensional case. As discussed in the introduction, the issue when looking for an effective equation is to obtain a well-posed equation. In particular, the sign of the tensor $a^{2}$ is crucial. We recall that a tensor $c \in \operatorname{Ten}^{4}\left(\mathbb{R}^{d}\right)$ is positive semidefinite if

$$
c\left(\eta \eta^{T}\right):\left(\eta \eta^{T}\right)=c_{i j k l} \eta_{i} \eta_{j} \eta_{k} \eta_{l} \geq 0 \quad \forall \eta \in \mathbb{R}^{d} .
$$

Let us investigate the signs of the tensors involved in the right hand side of (4.4c). First, the tensor $\left\langle a_{j k} \chi_{l} \chi_{i}\right\rangle_{Y}-\left\langle a \nabla \theta_{j i} \cdot \nabla \theta_{k l}\right\rangle_{Y}-a_{j k}^{0}\left\langle\chi_{l} \chi_{i}\right\rangle_{Y}$ is known to be negative semidefinite (this is shown in Ref. 12 in the framework of the Bloch wave theory see also Ref. 11, 14 and Section $5)$. Second, note that $\left\langle\chi_{i} \chi_{j}\right\rangle_{Y}$ is a symmetric positive semidefinite matrix. However, as the tensor $q_{i j k l}=\left\langle a_{j k} \chi_{l} \chi_{i}\right\rangle_{Y}-\left\langle a \nabla \theta_{j i} \cdot \nabla \theta_{k l}\right\rangle_{Y}$ has no sign (see e.g. Ref. 5), the pair $b^{0}=\left\langle\chi_{i} \chi_{j}\right\rangle_{Y}$, $a_{i j k l}^{2}=q_{i j k l}$ does not satisfy the requirements (4.4). In fact, to construct a valid pair of effective tensors $a^{2}, b^{0}$, we need to use the "freedom" provided by the minus sign in the constraint (4.4c). Indeed, we verify that if $R \in \operatorname{Sym}^{2}\left(\mathbb{R}^{d}\right)$ is positive definite, then the tensor $a_{i j}^{0} R_{k l}$ is positive definite

$$
a_{i j}^{0} R_{k l} \eta_{i} \eta_{j} \eta_{k} \eta_{l}=\left(\eta^{T} a^{0} \eta\right)\left(\eta^{T} R \eta\right) \quad \forall \eta \in \mathbb{R}^{d}
$$

where $a^{0}$ is positive definite. Hence, if we consider a sequence of parametrized positive definite matrices $\left\{R^{r}\right\}_{r>0} \subset \operatorname{Sym}^{2}\left(\mathbb{R}^{d}\right)$, such that the smallest eigenvalue of $R^{r}$ increases as $r$ increases, then, for sufficiently large values of $r$, the tensors

$$
a_{i j k l}^{2}=\left\langle a_{j k} \chi_{i} \chi_{l}\right\rangle_{Y}-\left\langle a \nabla \theta_{j i} \cdot \nabla \theta_{k l}\right\rangle_{Y}+a_{j k}^{0} R_{i l}^{r}, \quad b_{i j}^{0}=\left\langle\chi_{i} \chi_{j}\right\rangle_{Y}+R_{i j}^{r},
$$

satisfy all the requirements (4.4). This construction proves that the family of effective equations $\mathcal{E}$, defined in Definition 3.1, is not empty (see Figure 2, Section 6).

We now need a process to construct a matrix $R^{r}$ with sufficiently large eigenvalues for $a^{2}$ in (4.6) to be positive semidefinite. For that purpose, we introduce the following concept of positivity for a fourth order tensor $c \in \operatorname{Ten}^{4}\left(\mathbb{R}^{d}\right)$ :

$$
c \xi: \xi=c_{i j k l} \xi_{i j} \xi_{k l} \geq 0 \quad \forall \xi \in \operatorname{Sym}^{2}\left(\mathbb{R}^{d}\right) .
$$

As $\eta \in \mathbb{R}^{d}$ satisfies $\eta \eta^{T} \in \operatorname{Sym}^{2}\left(\mathbb{R}^{d}\right)$, assertion (4.7) implies (4.5). The advantage of (4.7) is that it can be seen as a simple eigenvalue problem. Indeed, consider the linear map $\operatorname{Sym}^{2}\left(\mathbb{R}^{d}\right) \rightarrow$ $\operatorname{Sym}^{2}\left(\mathbb{R}^{d}\right), \xi \mapsto c \xi$ defined by $(c \xi)_{i j}=c_{i j k l} \xi_{k l}$ and note $M(c)$ the associated $N(d) \times N(d)$ matrix, where $N(d)=\left(\begin{array}{c}d+1 \\ 2\end{array}\right)$. Then, we can construct a bijective map $\nu: \operatorname{Sym}^{2}\left(\mathbb{R}^{d}\right) \rightarrow \mathbb{R}^{N(d)}$ such that

$$
c \xi: \xi=M(c) \nu(\xi) \cdot \nu(\xi) \quad \forall \xi \in \operatorname{Sym}^{2}\left(\mathbb{R}^{d}\right) .
$$

Hence, the tensor $c$ satisfies (4.7) if and only if the matrix $M(c)$ is positive semidefinite and similarly, $c \xi: \xi>0 \forall \xi \in \operatorname{Sym}^{2}\left(\mathbb{R}^{d}\right) \backslash\{0\}$ if and only if $M(c)$ is positive definite. In Appendix A, we give the details on one possible constructions for $M(c)$ and $\nu$.

Now, we still need to ensure that increasing $r$ in (4.6) increases the eigenvalues of $M\left(a^{2}\right)$. This is proved by the following lemma.

Lemma 4.1. Let $A, R \in \operatorname{Sym}^{2}\left(\mathbb{R}^{d}\right)$ be positive definite matrices. Then, the tensor $c_{i j k l}=$ $A_{j k} R_{i l}$ satisfies $c \xi: \xi>0 \forall \xi \in \operatorname{Sym}^{2}\left(\mathbb{R}^{d}\right) \backslash\{0\}$. 
Proof. As $R$ is symmetric positive definite, the Cholesky factorization ensures the existence of an invertible matrix $H \in \operatorname{Ten}^{2}\left(\mathbb{R}^{d}\right)$ such that $R=H^{T} H$. As $A$ is positive definite, for $\xi \in \operatorname{Sym}^{2}\left(\mathbb{R}^{d}\right)$ we have

$$
c \xi: \xi=A_{j k} R_{i l} \xi_{i j} \xi_{k l}=A_{j k}\left(H_{m i} \xi_{i j}\right)\left(H_{m l} \xi_{l k}\right)=\left(\xi H_{m}\right)^{T} A \xi H_{m} \geq 0,
$$

where we denoted $H_{m}=\left(H_{m 1}, \ldots, H_{m d}\right)^{T}$. Now, assume that the equality holds. Then, as $A$ is positive definite it must hold $\xi H_{m}=0$ for all $m=1, \ldots, d$ or equivalently $\xi H^{T}=0$. As $H$ is regular so is $H^{T}$ and we conclude that $\xi=0$. The proof of the lemma is complete.

We now have a constructive method to obtain effective equations. Indeed, in the following lemma, we consider (4.6) with $R^{r}=r I$ (note that we could use $R^{r}=r a^{0}$ as well).

Lemma 4.2. Let $\tilde{a}_{i j k l}^{2}=\left\langle a_{j k} \chi_{i} \chi_{l}\right\rangle_{Y}-\left\langle a \nabla \theta_{j i} \cdot \nabla \theta_{k l}\right\rangle_{Y}$ and denote $A^{2}=M\left(\tilde{a}^{2}\right), A^{0}=$ $M\left(\left\{a_{j k}^{0} \delta_{i l}\right\}\right)$ and their respective minimal eigenvalues $\lambda_{\min }\left(A^{2}\right)$ and $\lambda_{\min }\left(A^{0}\right)$. Then, the tensor

$$
a_{i j k l}^{2}=\tilde{a}_{i j k l}^{2}+r a_{j k}^{0} \delta_{i l}, \quad \forall r \geq r^{*}=\max \left\{0,-\frac{\lambda_{\min }\left(A^{2}\right)}{\lambda_{\min }\left(A^{0}\right)}\right\},
$$

satisfies $a^{2} \xi: \xi \geq 0 \quad \forall \xi \in \operatorname{Sym}^{2}\left(\mathbb{R}^{d}\right)$.

Proof. First, as $A^{2}$ and $A^{0}$ are symmetric matrices it is clear that $\lambda_{\min }\left(A^{2}\right)$ and $\lambda_{\min }\left(A^{0}\right)$ are real and thanks to Lemma 4.1 and (4.8) it holds $\lambda_{\min }\left(A^{0}\right)>0$. Furthermore, $\lambda_{\min }\left(A^{2}\right) \leq$ $\left(A^{2} v \cdot v\right) /(v \cdot v)$ for any $v \in \mathbb{R}^{N(d)}$ and similarly for $A^{0}$. Now, if $A^{2}$ is positive semidefinite, then $r^{*}=0$ and the tensor $a^{2}$ is positive semidefinite for any $r \geq 0$. Next, assume that $\lambda_{\min }\left(A^{2}\right)<0$. We verify then that for any $v \in \mathbb{R}^{N(d)}$,

$$
r^{*}=-\frac{\lambda_{\min }\left(A^{2}\right)}{\lambda_{\min }\left(A^{0}\right)} \geq-\frac{A^{2} v \cdot v}{A^{0} v \cdot v} .
$$

Hence, for $\xi \in \operatorname{Sym}^{2}\left(\mathbb{R}^{d}\right)$ we note $v=\nu(\xi)$ (see (4.8)), set $r=r^{*}+\Delta r$ with $\Delta r \geq 0$ and obtain

$$
a^{2} \xi: \xi=A^{2} v \cdot v+r^{*} A^{0} v \cdot v+\Delta r A^{0} v \cdot v \geq 0 .
$$

The proof of the lemma is complete.

\section{Algorithm to compute the coefficients of an effective equation}

As discussed in the previous section, Theorem 3.1 and Lemma 4.2 give a way to construct an effective equation. We give here the full algorithm to compute the effective tensors $a^{0}, b^{0}$ and $a^{2}$. This algorithm is appropriate for dimensions $d \geq 2$ as a much simpler one can be obtained for $d=1$.

In order to state the algorithm in an optimal way, let us make an observation. We first introduce two sets of indices. Let $I(d) \subset\{1, \ldots, s\}^{4}$ be the set of indices of distinct entries of a tensor in $\operatorname{Sym}^{4}\left(\mathbb{R}^{d}\right)$. In particular, $|I(d)|=\left(\begin{array}{c}d+3 \\ 4\end{array}\right)$ so that $|I(2)|=5$ and $|I(3)|=15$. Next, a tensor $c \in \operatorname{Ten}^{4}\left(\mathbb{R}^{d}\right)$ is said to satisfies the major and minor symmetries if $c_{i j k l}=c_{l k j i}$ and $c_{i j k l}=c_{j i k l}=c_{i j l k}$ for all $1 \leq i, j, k, l \leq d$, respectively. We denote $J(d) \subset\{1, \ldots, s\}^{4}$ the set of indices of distinct entries of a tensor satisfying the major and minor symmetries. In particular, $|J(d)|=\left(\begin{array}{c}N(d)+1 \\ 2\end{array}\right)$ where $N(d)=\left(\begin{array}{c}d+1 \\ 2\end{array}\right)$ so that $|J(2)|=6$ and $|J(3)|=21$. Now, note that to compute the operator $a_{i j k l}^{2} \partial_{i j k l}^{4}$ we only need the entries $\left\{a_{i j k l}^{2}:(i, j, k, l) \in I(d)\right\}$. Furthermore, to construct the matrix $A^{2}$ from Lemma 4.2 associated to the tensor $\tilde{a}^{2}$, we only need the entries $\left\{\bar{a}_{i j k l}^{2}=\frac{1}{4}\left(\tilde{a}_{i j k l}^{2}+\tilde{a}_{j i k l}^{2}+\tilde{a}_{i j l k}^{2}+\tilde{a}_{j i l k}^{2}\right):(i, j, k, l) \in J(d)\right\}$. The conclusion is that to compute the coefficients of $a_{i j k l}^{2} \partial_{i j k l}^{4}$, computing the $|J(d)|$ distinct entries of $\bar{a}_{i j k l}^{2}$ is sufficient.

We are now ready to give the algorithm to compute the effective quantities $a^{0}, b^{0}, a^{2}$ of an effective equation in the family $\mathcal{E}$. 
Algorithm 1.

(1) for $1 \leq k \leq d$ find $\chi_{k} \in \mathrm{W}_{\text {per }}(Y)$ such that $\forall w \in \mathrm{W}_{\text {per }}(Y)$

$\left(a \nabla \chi_{k}, \nabla w\right)_{Y}=-\left(a e_{k}, \nabla w\right)_{Y}$,

(2) for $1 \leq i \leq j \leq d$ compute

$a_{i j}^{0}=a_{j i}^{0}=\left\langle a_{i j}+e_{i}^{T} a \nabla \chi_{j}\right\rangle_{Y}$

(3) for $1 \leq i \leq j \leq d$ find $\theta_{i j}=\theta_{j i} \in \mathrm{W}_{\text {per }}(Y)$ such that $\forall w \in \mathrm{W}_{\text {per }}(Y)$

$\left(a \nabla \theta_{i j}, \nabla w\right)_{Y}=-\frac{1}{2}\left(a\left(\chi_{i} e_{j}+\chi_{j} e_{i}\right), \nabla w\right)_{Y}+\frac{1}{2}\left(e_{i}^{T} a \nabla \chi_{j}+e_{j}^{T} a \nabla \chi_{i}, w\right)_{Y}+\left(a_{i j}-a_{i j}^{0}, w\right)_{Y}$,

(4) for $(i, j, k, l) \in J(d)$ compute

$\bar{a}_{i j k l}^{2}=\frac{1}{4}\left\langle a\left(\chi_{i} e_{j}+\chi_{j} e_{i}\right) \cdot\left(\chi_{l} e_{k}+\chi_{k} e_{l}\right)\right\rangle_{Y}-\left\langle a \nabla \theta_{i j} \cdot \nabla \theta_{k l}\right\rangle_{Y}$,

(5) build the matrices $A^{2}=M\left(\bar{a}^{2}\right), A^{0}=M\left(\left\{a_{j k}^{0} \delta_{i l}\right\}\right)$ (see Lemma Appendix A.1) and set

$r^{*}=\max \left\{0,-\frac{\lambda_{\min }\left(A^{2}\right)}{\lambda_{\min }\left(A^{0}\right)}\right\}$,

(6) for $1 \leq i \leq j \leq d$ compute

$b_{i j}^{0}=b_{j i}^{0}=\left\langle\chi_{i} \chi_{j}\right\rangle_{Y}+r^{*} \delta_{i j}$,

(7) for $(i, j, k, l) \in J(d)$ compute

$a_{i j k l}^{2}=\bar{a}_{i j k l}^{2}+r^{*} \frac{1}{4}\left(a_{j k}^{0} \delta_{i l}+a_{i k}^{0} \delta_{j l}+a_{j l}^{0} \delta_{i k}+a_{i l}^{0} \delta_{j k}\right)$.

\section{Comparison with the coefficients obtained via Bloch wave expansion}

We have seen in Section 2.2 how to compute the effective coefficients using asymptotic expansion. Yet we mentioned in the introduction that the existing effective model uses the expansion of $u^{\varepsilon}$ in Bloch waves. This approach has been used in a formal way by Santosa and Symes Ref. 22 and led to the rigorous well-posed effective model obtained by Dohnal, Lamacz and Schweizer in Ref. 14, 15. Note that it has also been widely used in the elliptic case (see Ref. 13 and the references therein). In this section, we compare the effective tensors obtained in this paper with the ones obtained in Ref. 14, 15. We show that the two approaches lead to similar cell problems and to the same tensors. Furthermore, we prove that the effective equation from Ref. 14, 15 belongs to the family of effective equations $\mathcal{E}$ defined in Definition 3.1. Note that, this comparison has recently been done in Ref. 5 , with a focus on the elliptic case.

Let us first summarize the result from Ref. 14, 15. The starting point is the expression of $u^{\varepsilon}$ in Bloch waves. We consider hence the solution $u^{\varepsilon}$ of equation (1.1) (with a zero right hand side) with the initial conditions $u^{\varepsilon}(0, x)=g(x), \partial_{t} u^{\varepsilon}(0, x)=0$. The $Y$-periodic symmetric tensor $a$ is assumed in $\left[\mathcal{C}^{1}(\bar{Y})\right]^{d \times d}$ and $g \in \mathrm{L}^{2}\left(\mathbb{R}^{d}\right) \cap \mathrm{L}^{1}\left(\mathbb{R}^{d}\right)$ is such that its Fourier transform $G$ has a compact support $K \subset \subset \mathbb{R}^{n}$. We fix the period $Y=(-\pi, \pi)^{d}$ and define the reciprocal periodicity cell $Z=(-1 / 2,1 / 2)^{d}$. Then, for a fixed $k \in Z$ we construct $\left\{\mu_{m}(k), \psi_{m}(y, k)\right\}_{m=0}^{\infty}$ the eigenvalues and eigenfunctions of the problem

$$
-\left(\nabla_{y}+\mathrm{i} k\right) \cdot\left(a(y)\left(\nabla_{y}+\mathrm{i} k\right) \psi_{m}(y, k)\right)=\mu_{m}(k) \psi_{m}(y, k),
$$

where $\mu_{m}(k)$ are real and $\mu_{m+1}(k) \geq \mu_{m}(k) \geq 0$. We define then the rescaled Bloch waves $w_{m}^{\varepsilon}(x, k)=\psi_{m}\left(\frac{x}{\varepsilon}, \varepsilon k\right) e^{i k \cdot x}$ and the rescaled eigenvalues $\mu_{m}^{\varepsilon}(k)=\mu_{m}(\varepsilon k)$. In particular, $\left\{\mu_{m}^{\varepsilon}(k), w_{m}^{\varepsilon}(x, k)\right\}$ satisfy

$$
-\nabla_{x} \cdot\left(a\left(\frac{x}{\varepsilon}\right) \nabla_{x} w_{m}^{\varepsilon}(x, k)\right)=\mu_{m}^{\varepsilon}(k) w_{m}^{\varepsilon}(x, k),
$$

and the Bloch waves $\left\{w_{m}^{\varepsilon}(x, k)\right\}_{m \geq 0}$ form a basis of $\mathrm{L}^{2}\left(\mathbb{R}^{d}\right)$. Then, $u^{\varepsilon}$ can be expressed as

$$
u^{\varepsilon}(t, x)=\sum_{m=0}^{\infty} \int_{Z / \varepsilon} \hat{g}_{m}^{\varepsilon}(k) w_{m}(x, k) \Re\left(e^{\mathrm{i} t \sqrt{\mu_{m}^{\varepsilon}(k)}}\right) \mathrm{d} k, \quad \hat{g}_{m}^{\varepsilon}(k)=\int_{\mathbb{R}^{d}} g(x) \overline{w_{m}^{\varepsilon}(x, k)} \mathrm{d} x,
$$

where $\bar{z}$ denotes the complex conjugate of $z$. First, it is proved in Ref. 14 that only the term with $m=1$ is relevant for the homogenization process. Then, the approximation

$$
U^{\varepsilon}(t, x)=(2 \pi)^{-d / 2} \int_{K} G(k) e^{\mathrm{i} k \cdot x} \Re\left(e^{\mathrm{i} t \sqrt{\mu_{0}^{\varepsilon}(k)}}\right) \mathrm{d} k,
$$


where $G$ is the Fourier transform of $g$ and $K$ is its support, is proved to satisfy the error estimate

$$
\left\|u^{\varepsilon}-U^{\varepsilon}\right\|_{\mathrm{L}^{\infty}\left(0, \infty ; \mathrm{L}^{2}\left(\mathbb{R}^{d}\right)+\mathrm{L}^{\infty}\left(\mathbb{R}^{d}\right)\right)} \leq C \varepsilon,
$$

where the norm $\|u\|_{\mathrm{L}^{2}\left(\mathbb{R}^{d}\right)+\mathrm{L}^{\infty}\left(\mathbb{R}^{d}\right)}=\inf \left\{\left\|u_{1}\right\|_{\mathrm{L}^{2}\left(\mathbb{R}^{d}\right)}+\left\|u_{2}\right\|_{\mathrm{L}^{\infty}\left(\mathbb{R}^{d}\right)}: u=u_{1}+u_{2}\right\}$ is weaker than both norms $\|\cdot\|_{\mathrm{L}^{2}\left(\mathbb{R}^{d}\right)}$ and $\|\cdot\|_{\mathrm{L}^{\infty}\left(\mathbb{R}^{d}\right)}$. The next step is the approximation of $\Re\left(e^{\mathrm{it} \sqrt{\mu_{0}^{\varepsilon}(k)}}\right)$ using Taylor expansion. Noting $\mu_{0}^{\varepsilon}(k)=A_{i j} k_{i} k_{j}+\varepsilon^{2} C_{i j m n} k_{i} k_{j} k_{m} k_{n}+\mathcal{O}\left(\varepsilon^{4}\right)$, where $A_{i j}=\partial_{i j}^{2} \mu_{0}(0)$ and $C_{i j m n}=\partial_{i j m n}^{4} \mu_{0}(0)$, we obtain the approximation

$$
v^{\varepsilon}(t, x)=(2 \pi)^{-d / 2} \frac{1}{2} \sum_{ \pm} \int_{K} G(k) e^{\mathrm{i} k \cdot x} \exp \left( \pm \mathrm{i} t \sqrt{A_{i j} k_{i} k_{j}}\right) \exp \left( \pm \frac{\mathrm{i} \varepsilon^{2} t}{2} \frac{C_{i j m n} k_{i} k_{j} k_{m} k_{n}}{\sqrt{A_{i j} k_{i} k_{j}}}\right) .
$$

The function $v^{\varepsilon}$ satisfies then the error estimate

$$
\left\|U^{\varepsilon}-v^{\varepsilon}\right\|_{\mathrm{L}^{\infty}\left(0, \varepsilon^{-2} T ; \mathrm{L}^{2}\left(\mathbb{R}^{d}\right)+\mathrm{L}^{\infty}\left(\mathbb{R}^{d}\right)\right)} \leq C \varepsilon .
$$

As shown in Ref. 15, it holds in fact $A_{i j}=\partial_{i j}^{2} \mu_{0}(0)=a_{i j}^{0}$, where $a^{0}$ is the homogenized tensor defined in (2.8). Hence, $v^{\varepsilon}$ satisfies

$$
\partial_{t}^{2} v^{\varepsilon}=a_{i j}^{0} \partial_{i j}^{2} v^{\varepsilon}-\varepsilon^{2} C_{i j m n} \partial_{i j m n}^{4} v^{\varepsilon}-\varepsilon^{4}\left(C_{i j m n} k_{i} k_{j} k_{m} k_{n}\right)^{2} /\left(4 a_{i j}^{0} k_{i} k_{j}\right) v^{\varepsilon} .
$$

However, $C$ being negative, the equation $\partial_{t}^{2} \cdot=a_{i j}^{0} \partial_{i j}^{2} \cdot-\varepsilon^{2} C_{i j m n} \partial_{i j m n}^{4}$. is ill-posed and cannot be used. An algebraic procedure is then applied in Ref. 14, 15 to build the tensors $E \in$ $\operatorname{Ten}^{2}\left(\mathbb{R}^{d}\right), F \in \operatorname{Ten}^{4}\left(\mathbb{R}^{d}\right)$, satisfying the symmetry and positivity (3.1), such that the following decomposition holds :

$$
-C_{i j m n} \partial_{i j m n}^{4}=E_{i j} \partial_{i j}^{2} a_{m n}^{0} \partial_{m n}^{2}-F_{i j m n} \partial_{i j m n}^{4}
$$

We observe that decomposition (5.4) is a preparation to a "Boussinesq trick", i.e., to use the effective equation to replace the operator $a_{m n}^{0} \partial_{m n}^{2}$ with $\partial_{t}^{2}$ (with a higher order error term). Then, it is proved that the solution $w^{\varepsilon}$ of the (well-posed) equation

$$
\begin{aligned}
& \partial_{t}^{2} w^{\varepsilon}=a_{i j}^{0} \partial_{i j}^{2} w^{\varepsilon}+\varepsilon^{2}\left(E_{i j} \partial_{i j}^{2} \partial_{t}^{2} w^{\varepsilon}-F_{i j m n} \partial_{i j m n}^{4} w^{\varepsilon}\right) \quad \text { in }\left(0, T^{\varepsilon}\right] \times \mathbb{R}^{d}, \\
& w^{\varepsilon}(0, x)=g(x), \quad \partial_{t} w^{\varepsilon}(0, x)=0,
\end{aligned}
$$

satisfies $\left\|\nabla\left(v^{\varepsilon}-w^{\varepsilon}\right)\right\|_{\mathrm{L}^{\infty}\left(0, T^{\varepsilon} ; \mathrm{L}^{2}\left(\mathbb{R}^{d}\right)\right)} \leq C \varepsilon^{2}$, which combined with the estimates (5.2), (5.3) allows to prove that $\left\|u^{\varepsilon}-w^{\varepsilon}\right\|_{\mathrm{L}^{\infty}\left(0, T^{\varepsilon} ; \mathrm{L}^{2}\left(\mathbb{R}^{d}\right)+\mathrm{L}^{\infty}\left(\mathbb{R}^{d}\right)\right)} \leq C \varepsilon$.

Let us now give the explicit formulas from Ref. 14 to compute $C_{i j k l}=\partial_{i j k l}^{4} \mu_{0}(0)$. We consider the following cell problems : for $1 \leq i \leq j \leq k \leq d$, find $\psi_{0}^{e_{j}}, \psi_{0}^{e_{i}+e_{j}}, \psi_{0}^{e_{i}+e_{j}+e_{k}}$ the $Y$-periodic zero mean solutions of

$$
\begin{aligned}
& -\nabla \cdot\left(a \nabla \psi_{0}^{e_{j}}\right)=\mathrm{i} \nabla \cdot\left(a e_{j}\right) \\
& -\nabla \cdot\left(a \nabla \psi_{0}^{e_{i}+e_{j}}\right)=2 S_{i j}^{2}\left\{\mathrm{i} \nabla \cdot\left(a e_{i} \psi_{0}^{e_{j}}\right)+\mathrm{i} e_{i}^{T} a \nabla \psi_{0}^{e_{j}}-a_{i j}+a_{i j}^{0}\right\} \\
& -\nabla \cdot\left(a \nabla \psi_{0}^{e_{i}+e_{j}+e_{k}}\right)=3 S_{i j k}^{3}\left\{\mathrm{i} \nabla \cdot\left(a e_{i} \psi_{0}^{e_{j}+e_{k}}\right)+\mathrm{i} e_{i}^{T} a \nabla \psi_{0}^{e_{j}+e_{k}}-2 a_{i j}(y) \psi_{0}^{e_{k}}+2 a_{i j}^{0} \psi_{0}^{e_{k}}\right\}
\end{aligned}
$$

Then, $C$ is given for $1 \leq i, j, k, l \leq d$ by

$$
C_{i j k l}=\frac{1}{2} S_{i j k l}^{4}\left\{\left\langle a_{i j} \psi_{0}^{e_{k}+e_{l}}\right\rangle_{Y}\right\}-\frac{1}{6} \mathrm{i} S_{i j k l}^{4}\left\{\left\langle e_{i}^{T} a \nabla \psi_{0}^{e_{j}+e_{k}+e_{l}}\right\rangle_{Y}\right\} .
$$

The cell problems $(5.5 \mathrm{a}),(5.5 \mathrm{~b})$ and $(5.5 \mathrm{c})$ are very similar to the ones we obtain in $(2.12 \mathrm{a})$, (2.12b) and (2.12c) with asymptotic expansion. Let us determine their exact relation. First, note that $\psi_{0}^{e_{j}}$ and $\psi_{0}^{e_{i}+e_{j}+e_{k}}$ are pure complex valued and $\psi_{0}^{e_{i}+e_{j}}$ are real valued (that ensures that $C_{i j k l}$ is real). Second, consider $\chi_{k}, \theta_{i j}, \kappa_{i j k}$ the zero mean solutions of respectively problems (2.12a), (2.12b) and (2.12c). Using the unicity of a solution of an elliptic boundary value problem, we see that

$$
\psi_{0}^{e_{j}}=\mathrm{i} \chi_{j}, \quad \psi_{0}^{e_{i}+e_{j}}=-2 \theta_{i j}, \quad \psi_{0}^{e_{i}+e_{j}+e_{k}}=-6 \mathrm{i} \kappa_{i j k} .
$$


We now show that the computed effective quantities are in fact exactly the same. Using (5.7), we rewrite $C_{i j k l}$ in (5.6) as

$$
C_{i j k l}=S_{i j k l}^{4}\left\{-\left\langle a_{i j} \theta_{k l}\right\rangle_{Y}-\left\langle e_{i}^{T} a \nabla \kappa_{j k l}\right\rangle_{Y}\right\}=|Y| S_{i j k l}^{4}\left\{-\left(a \nabla \kappa_{j k l}, e_{i}\right)_{Y}-\left(e_{j} \theta_{k l}, e_{i}\right)_{Y}\right\} .
$$

As $\left\langle\theta_{k l}\right\rangle_{Y}=0$, this expression is equal to the right hand side of (2.15). Hence, from (2.15) we have

$$
C_{i j k l}=S_{i j k l}^{4}\left\{\left\langle a_{j k} \chi_{l} \chi_{i}\right\rangle_{Y}-\left\langle a \nabla \theta_{j i} \cdot \nabla \theta_{k l}\right\rangle_{Y}-a_{j k}^{0}\left(\chi_{l}, \chi_{i}\right)_{Y}\right\} .
$$

Now, as $E, F$ defined in Ref. 15 satisfies (5.4), thanks to (5.8) we can infer that they satisfy the constraint (3.4) from Theorem 3.1. As $E, F$ satisfy by construction (3.1), the effective equation defined in Ref. 15 belongs to the family $\mathcal{E}$ defined in Definition 3.1.

Let us make a final remark. If we perform the asymptotic expansion as in Section 2.2 with the ansatz that the effective equation is of the form $\partial_{t}^{2} \tilde{u}-a_{i j}^{0} \partial_{i j}^{2} \tilde{u}+\varepsilon^{2} c_{i j k l} \partial_{i j k l}^{4} \tilde{u}=0$ (instead of (2.7)), we obtain on $c$ the constraint $S_{i j k l}^{4}\left\{c_{i j k l}\right\}=S_{i j k l}^{4}\left\{C_{i j k l}\right\}$. Hence, we end up with the operator $c_{i j k l} \partial_{i j k l}^{4}=C_{i j k l} \partial_{i j k l}^{4}$ and thus the same ill-posed equation as obtained in the first place in Ref. 22. As it happens here, there is no possible remedy to the non positivity of $c$. The conclusion is that when performing asymptotic expansion, the form of the effective equation that we postulate is a crucial ansatz.

\section{Numerical experiments}

Let $Y=(-1 / 2,1 / 2)$ and consider the $Y$-periodic diagonal tensor given by

$$
a_{11}(y)=a_{22}(y)=\tilde{a}\left(y_{2}\right)=1-0.5 \cos \left(2 \pi y_{2}\right), \quad a_{12}(y)=a_{21}(y)=0 .
$$

The oscillatory tensor $a\left(\frac{x}{\varepsilon}\right)$ describes a layered material and it is well known (see Ref. 7, 18, 10) that the homogenized tensor is given by

$$
a_{11}^{0}=\int_{-1 / 2}^{1 / 2} \tilde{a}\left(y_{2}\right) \mathrm{d} y_{2}=1, \quad a_{22}^{0}=\left(\int_{-1 / 2}^{1 / 2}\left(\tilde{a}\left(y_{2}\right)\right)^{-1} \mathrm{~d} y_{2}\right)^{-1}=\sqrt{3} / 2,
$$

and $a_{12}^{0}=a_{21}^{0}=0$. Furthermore, we have an analytic expression for the first correctors $\chi_{1}, \chi_{2}$. Nevertheless, in order to test the numerical procedure (Algorithm 1), the cell functions $\chi_{1}, \chi_{2}, \theta_{11}, \theta_{12}, \theta_{22}$ are computed with a P1 finite element method on a uniform mesh of $Y$ with 1024 points in both directions. We verify that we obtain a very accurate approximation of $a^{0}$. Then, we compute accurately the 6 distinct entries of the tensor $\bar{a}^{2}$ and find

$$
\begin{array}{lll}
\bar{a}_{1111}^{2}=-0.00339360, & \bar{a}_{2222}^{2}=0, & \bar{a}_{1212}^{2}=0.00086375, \\
\bar{a}_{1122}^{2}=0.00339360, & \bar{a}_{1112}^{2}=0, & \bar{a}_{2212}^{2}=0 .
\end{array}
$$

We construct then the $3 \times 3$ symmetric matrix $A^{2}=M\left(\bar{a}^{2}\right)$ and compute its eigenvalues as $\operatorname{spec}\left(A^{2}\right)=\{-0.0054909,0.0020973,0.0034550\}$. so that the matrix $A^{2}$ is not positive semidefinite. Hence, in order to compute the positive tensor $a^{2}$, we build the ma$\operatorname{trix} A^{0}=M\left(\left\{a_{j k}^{0} \delta_{i l}\right\}\right.$ ) (observe that $A^{0}$ is diagonal because $a^{0}$ is diagonal) and obtain $\operatorname{spec}\left(A^{0}\right)=\left\{a_{11}^{0}, a_{22}^{0}, a_{11}^{0}+a_{22}^{0}\right\}$. We then compute $r^{*}=\max \left\{0,-\frac{\lambda_{\min }\left(A^{2}\right)}{\lambda_{\min }\left(A^{0}\right)}\right\}=0.006340411$, the tensors $b^{0}, a^{2}$ are

$$
\begin{array}{ll} 
& a_{1111}^{2}=\bar{a}_{1111}^{2}+r^{*} a_{11}^{0}=0.00294681, \\
b_{11}^{0}=\left\langle\chi_{1}^{2}\right\rangle_{Y}+r^{*}=0.00634041, & a_{2222}^{2}=\bar{a}_{2222}^{2}+r^{*} a_{22}^{0}=0.00549097, \\
b_{22}^{0}=\left\langle\chi_{2}^{2}\right\rangle_{Y}+r^{*}=0.01004512, & a_{1212}^{2}=\bar{a}_{1212}^{2}+\frac{1}{4} r^{*}\left(a_{11}^{0}+a_{22}^{0}\right)=0.0038215948, \\
b_{12}^{0}=0, & a_{1122}^{2}=\bar{a}_{1122}^{2}=0.0033935973, \\
& a_{1112}^{2}=\bar{a}_{1112}^{2}+\frac{1}{4} r^{*}\left(a_{12}^{0}+a_{21}^{0}\right)=0, \\
& a_{2212}^{2}=\bar{a}_{1222}^{2}+\frac{1}{4}\left(a_{12}^{0}+a_{21}^{0}\right)=0,
\end{array}
$$

and we obtain the corresponding effective differential operators $b_{i j}^{0} \partial_{i j}^{2}, a_{i j k l}^{2} \partial_{i j k l}^{4}$.

We recall that other effective equations can be obtained by defining the tensors as in (4.6), where $R^{r} \in \operatorname{Sym}^{2}\left(\mathbb{R}^{d}\right)$ is a positive definite matrix with sufficiently large eigenvalues. In order 
to illustrate that, we let $r=\left(r_{1}, r_{2}\right) \in \mathbb{R}^{2}, R^{r}=\operatorname{diag}\left(r_{1}, r_{2}\right)$, and denote $a_{r}^{2}, b_{r}^{0}$ as defined in (4.6), where the subscript specify the dependence in $r$. For several values of $r$, we compute the minimal eigenvalue $\lambda_{\min }(r)$ of $M\left(a_{r}^{2}\right)$. In Figure 2, we plot $r=\left(r_{1}, r_{2}\right)$ with a red square ( $\left.\square\right)$ if $\lambda_{\min }(r)<0$ and a green square $(\square)$ if $\lambda_{\min }(r) \geq 0$. Hence, each green square corresponds to a different well-posed effective equation in the family and we call the corresponding $r$ valid. We observe that there is a distinct frontier between valid and invalid values of $r$. The black square is $\left(r^{*}, r^{*}\right)$, where $r^{*}$ is obtained in Lemma 4.2 and Algorithm 1. As expected, $\left(r^{*}, r^{*}\right)$ lies in the domain of valid values. The subset of the diagonal in the valid values $\left\{(r, r): r \geq r^{*}\right\}$ corresponds to the effective equations obtained using Lemma 4.2. In what follows, we denote by $\tilde{u}_{r}$ the solution of the effective equation (3.2) with $a^{2}=a_{r}^{2}, b^{0}=b_{r}^{0}$, for $r \geq r^{*}$. The effective solution given by Algorithm 1 is denoted by $\bar{u}=\tilde{u}_{r^{*}}$.

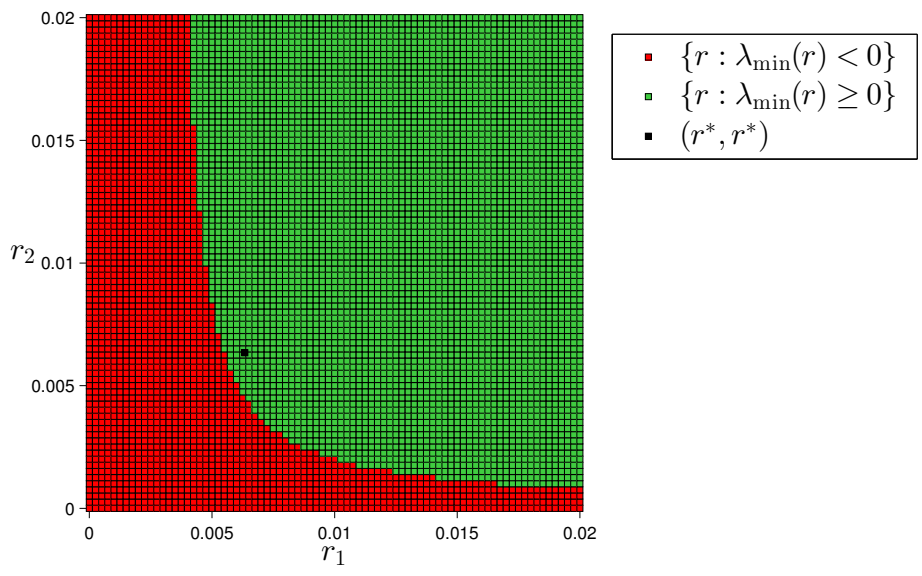

Fig. 2. Sorting of the minimal eigenvalues of $M\left(a_{r}^{2}\right)$, where $a_{r}^{2}$ is defined in (4.6) with $R^{r}=\operatorname{diag}\left(r_{1}, r_{2}\right)$. Each green square corresponds to an effective equation in the family $\mathcal{E}$. The black square is $\left(r^{*}, r^{*}\right)$, where $r^{*}$ is computed in Algorithm 1.

Let us now consider the model problem given by the initial conditions and source term

$$
\varepsilon=1 / 10, \quad g^{0}(x)=e^{-\frac{x_{1}^{2}+x_{2}^{2}}{1 / 5}}, \quad g^{1}(x)=0, \quad f(t, x)=0 .
$$

Let us describe how to approximate the homogenized solution $u^{0}(1.2)$ and the solution $\tilde{u}$ of the effective equation (3.2) for the data of the model problem (6.2) . Both equations involves constant coefficients differential operators and hence we have an explicit form of the solution with Fourier transform (see for example Ref. 17). Let us denote $\mathcal{F}(\cdot)$ and $\mathcal{F}^{-1}(\cdot)$ the (normalized) Fourier transform and its (normalized) inverse. Then, we verify that the solution of (3.2) is given for every time $t$ by

$$
\tilde{u}(t)=\mathcal{F}^{-1}\left(\mathcal{F}\left(g^{0}\right) \cos (\sqrt{s(k)} t)\right), \quad s(k)=\frac{a^{0} k \cdot k+\varepsilon^{2} a^{2} k k^{T}: k k^{T}}{1+\varepsilon^{2} b^{0} k \cdot k} .
$$

Similarly, the homogenized solution is obtained by replacing $s(k)$ with $a^{0} k \cdot k$ in $(6.3)$. Thus, $u^{0}(t), \tilde{u}_{r}(t)$ can be approximated very accurately. We approximate the Fourier transform and its inverse on a uniform grid using the FFTW library (C library for computing the discrete Fourier transforms using fast Fourier transform (FFT) algorithms). Note that Matlab's native FFT implementation (which is based on FFTW) can also be used.

First, we consider the small periodic domain $\Omega=(-2,2)^{2}$. On such a small domain we are able to approximate $u^{\varepsilon}$, the solution of (1.9) $\left(g^{0}\right.$ must be replaced with $g^{0}-\left\langle g^{0}\right\rangle_{\Omega}$ to fit the settings of (1.9)). To do so, we consider a uniform grid of $\Omega$ of size $h=\varepsilon / 10$ and use a pseudo spectral method (see for example Ref. 23 for an introduction) using the FFTW library. The time integration of the obtained second order ordinary differential equation is 
done with the leap frog scheme with a small time step $\Delta t=h / 100$. The solutions $u^{0}$ and $\bar{u}$ are approximated as described previously on the same grid as $u^{\varepsilon}$. We compute the relative errors $\left\|\left(u^{\varepsilon}-u^{0}\right)(t)\right\|_{\mathrm{L}^{2}(\Omega)} /\left\|u^{\varepsilon}(t)\right\|_{\mathrm{L}^{2}(\Omega)}$ and $\left\|\left(u^{\varepsilon}-\bar{u}\right)(t)\right\|_{\mathrm{L}^{2}(\Omega)} /\left\|u^{\varepsilon}(t)\right\|_{\mathrm{L}^{2}(\Omega)}$ on the time interval $[0,200]$. The result is displayed in Figure 3. We observe that the homogenized solution quickly drift away from the fine scale solution $u^{\varepsilon}$. As we know, this is due to the dispersion effects that develop in $u^{\varepsilon}$. On the contrary, we see that for times $\mathcal{O}\left(\varepsilon^{-2}\right)$ the error $u^{\varepsilon}-\bar{u}$ is very small, as predicted by Theorem 3.1.

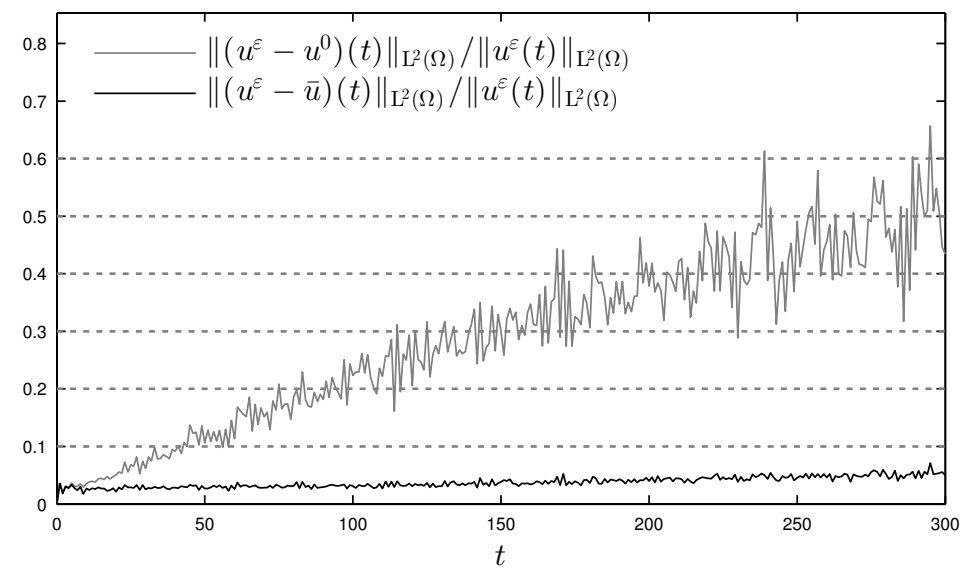

Fig. 3. Plot of the time evolution of the normalized $L^{2}(\Omega)$ errors $u^{\varepsilon}-u^{0}$ and $u^{\varepsilon}-\bar{u}$.

Let us now consider the wave equation with the settings (6.2) in the unbounded case. As the homogenized tensor (6.1) is diagonal, we know the form of the homogenized solution $u^{0}$ : the pulse $g^{0}$ centered at the origin spreads in all directions with speeds $\sqrt{a_{11}^{0}}$ along the $x$ axis and $\sqrt{a_{22}^{0}}$ along the $y$ axis. To obtain $\bar{u}(t)$ at a time $t$, we thus apply the formula (6.3) and approximate the Fourier transforms on a periodic truncation $\mathbb{R}^{2}$ given by $\Omega=$ $\left(-L_{1}, L_{1}\right) \times\left(-L_{2}, L_{2}\right)$, where $L_{i}=\sqrt{a_{i i}^{0}} t+R_{i}$ and $R_{i}>0$ is large enough $\left(R_{i}=4\right.$ in the experiment). We proceed similarly to approximate $u^{0}(t)$. In Figure 4 is displayed the global form of $\bar{u}$ at $t=300$ and in the zooms we can see the dispersion effects. Note that although $a\left(\frac{x}{\varepsilon}\right)$ oscillates only in the $y$ direction, the dispersion is as strong in the $x$ direction as in the $y$ direction. In the top-left plot of Figure 5 , we can see a $3 \mathrm{D}$ view of the dispersion of $\bar{u}$ on a small domain. Furthermore, the same view of $u^{0}$ is displayed in the top-right plot of Figure 5 and we see that there is no dispersion after the main pulse. In the bottom plot of Figure 5 , we can compare cuts at $y=0$ of $\bar{u},\left\{\tilde{u}_{r}\right\}_{r}$ for several values of $r \in\left[r^{*}, 11 r^{*}\right]$ and $u^{0}$. We see that all the effective solution $\left\{\tilde{u}_{r}\right\}_{r}$ and $\bar{u}$ have almost the same dispersive behavior. As Theorem 3.1 ensures that $\bar{u}$ and $\tilde{u}_{r}$ approximate well $u^{\varepsilon}$ in the $\mathrm{L}^{\infty}\left(\mathrm{L}^{2}\right)$ norm, we conclude that $u^{0}$ is a poor approximation of $u^{\varepsilon}$ at $t=300$.

Acknowledgements. This work was partially supported by the Fonds National Suisse, project No. 200021_150019.

\section{Appendix A. Matrix associated to a major symmetric tensor of order 4}

Let $a \in \operatorname{Ten}^{4}\left(\mathbb{R}^{d}\right)$ be a tensor of order 4 satisfying the major symmetry relation, i.e. $a_{i j k l}=a_{l k j i}$ $1 \leq i, j, k, l \leq d$. We say that $a$ is positive definite (resp. semidefinite) if $a \xi: \xi>0$ for any $\xi \in \operatorname{Sym}^{2}\left(\mathbb{R}^{d}\right) \backslash\{0\}$ (resp. $\geq 0$ ). In what follows, we define a matrix $M(a)$ such that $a$ is positive (semi)definite if and only if $M(a)$ is positive (semi)definite.

Without loss of generality, we can assume that $a$ satisfies the minor symmetry relations

$$
a_{i j k l}=a_{j i k l}=a_{i j l k} \quad 1 \leq i, j, k, l \leq d .
$$




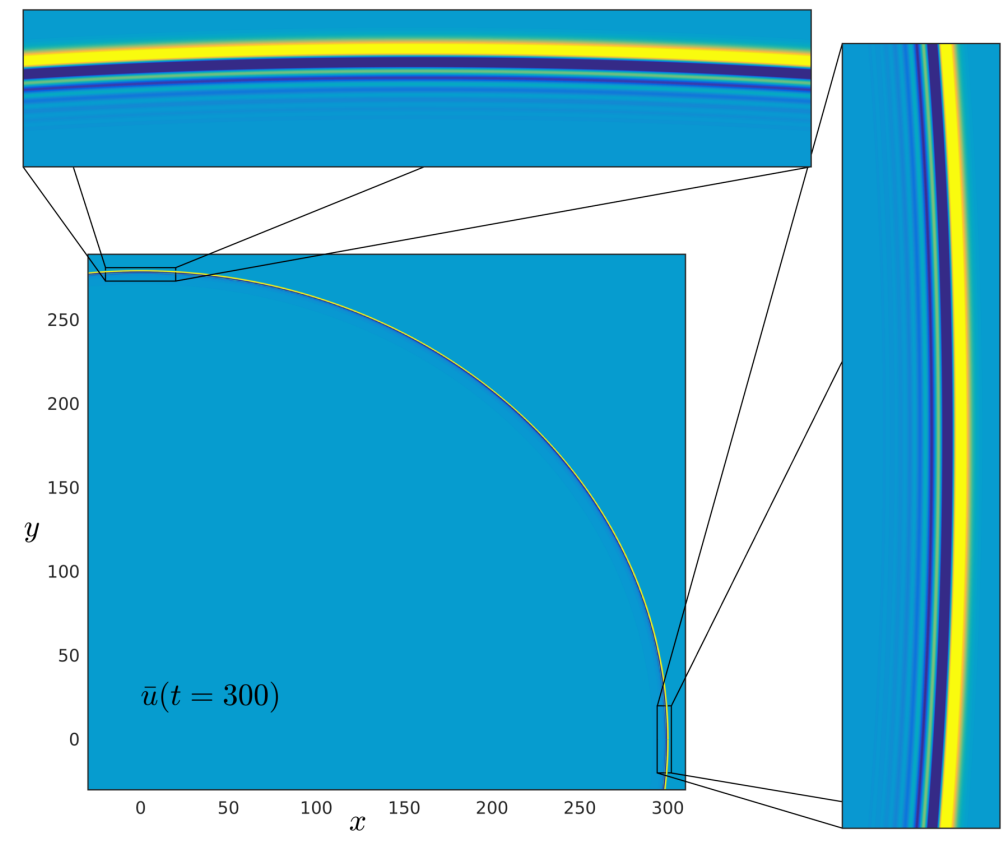

Fig. 4. Global view of $\bar{u}$ at $t=300$ and zooms on the subdomains $(294,302) \times(-20,20)$ and $(-20,20) \times(273,281)$.
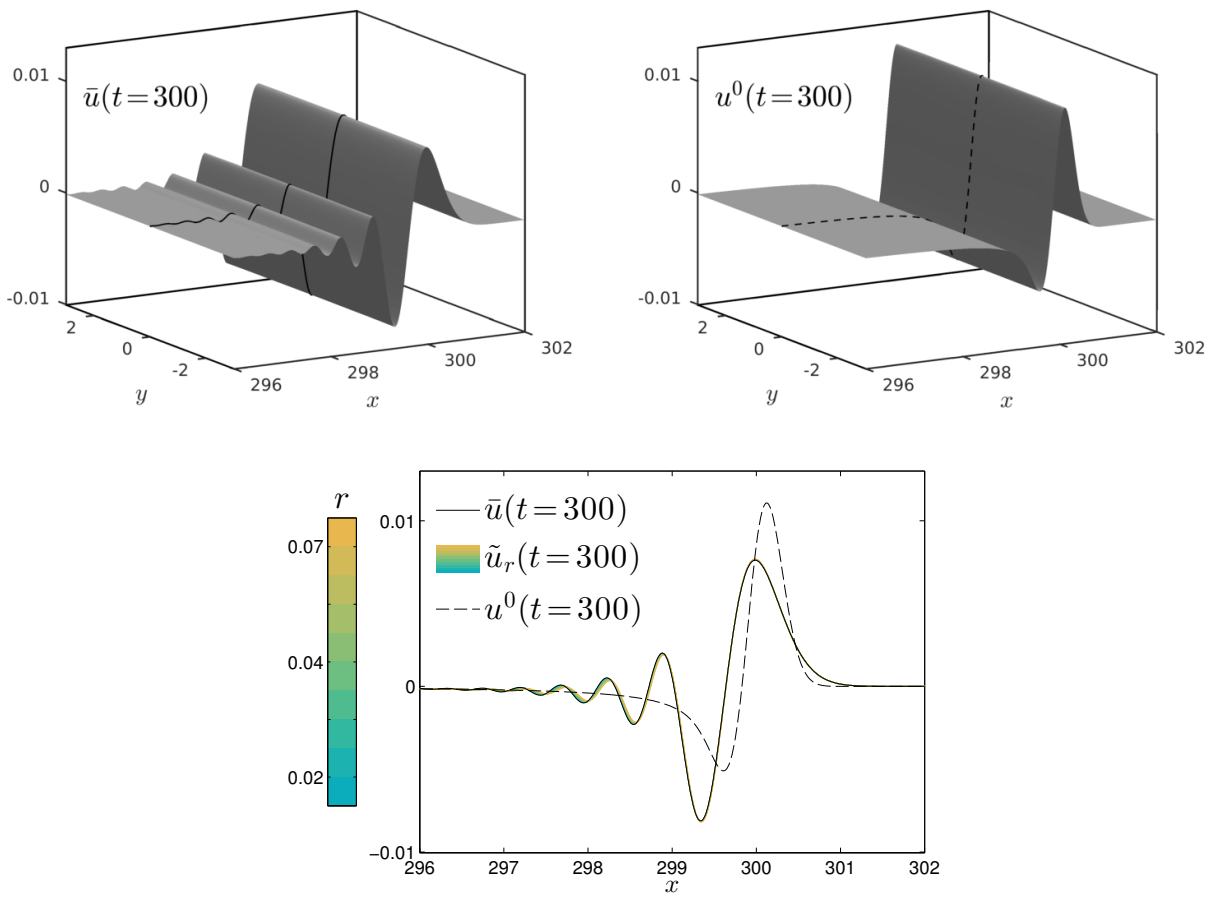

Fig. 5. Top : 3d views of $\bar{u}$ (top-left) and $u^{0}$ (top-right) at $t=300$ for $(x, y) \in(296,302) \times(-3,3)$ Bottom : cuts through $y=0$ at $t=300$ of $u^{0}$ and the effective solutions $\bar{u}, \tilde{u}_{r}$ for several values of $r \in\left[r^{*}, 11 r^{*}\right]$.

Indeed, if $a \in \operatorname{Ten}^{4}\left(\mathbb{R}^{d}\right)$ does not satisfy the minor symmetries (A.1), then $a_{i j k l}$ can be replaced by $\bar{a}_{i j k l}=\frac{1}{4}\left(a_{i j k l}+a_{j i k l}+a_{i j l k}+a_{j i l k}\right)$ which satisfies (A.1) and $a \xi: \eta=\bar{a} \xi: \eta$ for any 
$\xi, \eta \in \operatorname{Sym}^{2}\left(\mathbb{R}^{d}\right)$. The tensor $a$ defines a linear map $\operatorname{Sym}^{2}\left(\mathbb{R}^{d}\right) \rightarrow \operatorname{Sym}^{2}\left(\mathbb{R}^{d}\right), \xi \mapsto a \xi$ as

$$
(a \xi)_{i j}=a_{i j k l} \xi_{k l}=\sum_{k=1}^{d} a_{i j k k} \xi_{k k}+2 \sum_{k=1}^{d} \sum_{l=k+1}^{d} a_{i j k l} \xi_{k l} .
$$

In order to build a matrix associated to this linear map, we define the sets of indices $J=$ $\{(i, j): 1 \leq i \leq j \leq d\}$ and $I=\{1, \ldots, N(d)\}$, where $N(d)=\left(\begin{array}{c}d+1 \\ 2\end{array}\right)$ is the number of distinct entries of a symmetric matrix in $\operatorname{Sym}^{2}\left(\mathbb{R}^{d}\right)$. Let $\ell^{-1}: J \rightarrow I$ be the one to one map given by $\ell^{-1}(i, j)=K_{i j}^{d}$, where $K^{d}$ is the symmetric $d \times d$ matrix given by (fill the diagonal, then successively the $d-1$ upper diagonal rows)

$$
K^{d}=\left(\begin{array}{ccccc}
1 & d+1 & \cdots & \cdots & 2 d-1 \\
& 2 & 2 d & \cdots & 3 d-3 \\
& & \ddots & \ddots & \vdots \\
& & \ddots & N(d) \\
& & & & d
\end{array}\right) .
$$

Define then the bijective map $\nu: \operatorname{Sym}^{2}\left(\mathbb{R}^{d}\right) \rightarrow \mathbb{R}^{N(d)}, \xi \rightarrow \nu(\xi)$, by $(\nu(\xi))_{m}=\xi_{\ell(m)}$ and note that its inverse is given for $v \in \mathbb{R}^{N(d)}$ by $\left(\nu^{-1}(v)\right)_{i j}=v_{\ell^{-1}(i, j)}$. The linear map associated to (A.2) is then given as $A: \mathbb{R}^{N(d)} \rightarrow \mathbb{R}^{N(d)}, A=\nu \circ a \circ \nu^{-1}$. We verify that for $v \in \mathbb{R}^{N(d)}$,

$$
(A v)_{m}=\sum_{k=1}^{d} a_{\ell(m) \ell(k)} v_{k}+2 \sum_{k=d+1}^{N(d)} a_{\ell(m) \ell(k)} v_{k} .
$$

Hence, noting $\left\{e_{i}\right\}_{i=1}^{N(d)}$ the canonical basis of $\mathbb{R}^{N(d)}$, the matrix associated to $A$ is given in the basis $\left\{e_{1}, \ldots e_{d}, \frac{1}{2} e_{d+1}, \ldots, \frac{1}{2} e_{N(d)}\right\}$ by $A_{m n}=a_{\ell(m) \ell(n)}$. We can then show that for any $\xi, \eta \in \operatorname{Sym}^{2}\left(\mathbb{R}^{d}\right)$, we have

$$
\begin{aligned}
a \xi: \eta & =\sum_{i k=1}^{d} a_{i i k k} \xi_{i i} \eta_{k k}+2\left(\sum_{\substack{i k=1 \\
k<l}}^{d} a_{i i k l} \xi_{i i} \eta_{k l}+\sum_{\substack{j k=1 \\
i<j}}^{d} a_{i j k k} \xi_{i j} \eta_{k k}\right)+4 \sum_{\substack{i<j \\
k<l}}^{d} a_{i j k l} \xi_{i j} \eta_{k l} \\
& =\nu(\xi)^{T} P^{T} A P \nu(\eta),
\end{aligned}
$$

where

$$
P_{m n}=\delta_{m n} z_{n}, \quad z_{n}=\left\{\begin{array}{l}
1 \text { if } 1 \leq n \leq d \\
2 \text { if } d+1 \leq n \leq N(d)
\end{array}\right.
$$

Hence, we define the matrix associated to $a$ as $\tilde{M}(a)=P^{T} A P$, given by

$$
(\tilde{M}(a))_{m n}=z_{m} z_{n} a_{\ell(m) \ell(n)} .
$$

For $d=2,3 \tilde{M}(a)$ is given respectively as

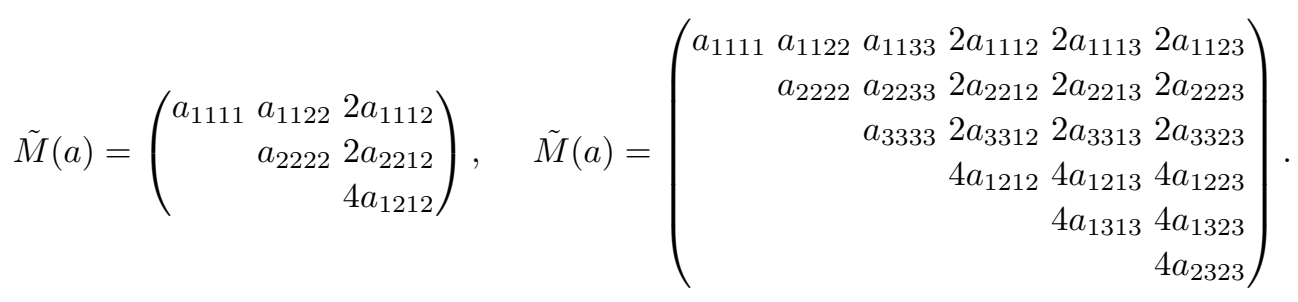

We summarize the results of this section in the following lemma.

Lemma Appendix A.1. Let $a \in \operatorname{Ten}^{4}\left(\mathbb{R}^{d}\right)$ be a tensor satisfying $a_{j i l k}=a_{l k j i}$ and define $\bar{a}_{i j k l}=\frac{1}{4}\left(a_{i j k l}+a_{j i k l}+a_{i j l k}+a_{j i l k}\right)$ and $M(a)=\tilde{M}(\bar{a})$, where $\tilde{M}$ is defined in (A.3). Then, $a$ is positive (semi)definite if and only if $M(a)$ is positive (semi)definite. 


\section{References}

1. A. Abdulle And M. J. Grote, Finite element heterogeneous multiscale method for the wave equation, Multiscale Model. Simul., 9 (2011), pp. 766-792.

2. A. Abdulle, M. J. Grote, And C. Stohrer, FE heterogeneous multiscale method for long-time wave propagation, C. R. Math. Acad. Sci. Paris, 351 (2013), pp. 495-499.

3. _ Finite element heterogeneous multiscale method for the wave equation: long-time effects, Multiscale Model. Simul., 12 (2014), pp. 1230-1257.

4. A. Abdulle And T. Pouchon, A priori error analysis of the finite element heterogenenous multiscale method for the wave equation in heterogeneous media over long time. submitted, 2015.

5. G. Allaire, M. Briane, and M. Vanninathan, A comparison between two-scale asymptotic expansions and bloch wave expansions for the homogenization of periodic structures, (2015). Preprint, hal-01215580.

6. D. Arjmand And O. Runborg, Analysis of heterogeneous multiscale methods for long time wave propagation problems, Multiscale Model. Simul., 12 (2014), pp. 1135-1166.

7. A. Bensoussan, J.-L. Lions, And G. Papanicolaou, Asymptotic analysis for periodic structures, North-Holland Publishing Co., Amsterdam, 1978.

8. L. Bers, F. John, And M. Schechter, Partial differential equations, vol. Proceedings of the summer seminar of Lectures in Applied Mathematics, Proceeding of the Summer Seminar, Boulder, CO, 1957.

9. S. Brahim-Otsmane, G. A. Francfort, and F. Murat, Correctors for the homogenization of the wave and heat equations, J. Math. Pures Appl., 71 (1992), pp. 197-231.

10. D. Cioranescu And P. Donato, An introduction to homogenization, vol. 17 of Oxford Lecture Series in Mathematics and its Applications, Oxford University Press, New York, 1999.

11. C. Conca, R. Orive, And M. Vanninathan, Bloch approximation in homogenization and applications, SIAM J. Math. Anal., 33 (2002), pp. 1166-1198.

12. - On burnett coefficients in periodic media, J. Math. Phys., 47 (2006).

13. C. Conca And M. Vanninathan, Homogenization of periodic structures via bloch decomposition, SIAM J. Appl. Math., 57 (1997), pp. 1639-1659.

14. T. Dohnal, A. Lamacz, And B. Schweizer, Bloch-wave homogenization on large time scales and dispersive effective wave equations, Multiscale Model. Simul., 12 (2014), pp. 488-513.

15. — Dispersive homogenized models and coefficient formulas for waves in general periodic media, Asymptot. Anal., 93 (2015), pp. 21-49.

16. B. Engquist, H. Holst, And O. Runborg, Multiscale methods for wave propagation in heterogeneous media over long time, in Numerical analysis of multiscale computations, Springer, 2012, pp. $167-186$.

17. L. C. Evans, Partial Differential Equations, Graduate studies in mathematics, American Mathematical Society, 1998.

18. V. V. Jikov, S. M. Kozlov, And O. A. Oleinik, Homogenization of differential operators and integral functionals, Springer-Verlag, Berlin, Heidelberg, 1994.

19. A. LAmaCZ, Dispersive effective models for waves in heterogeneous media, Math. Models Methods Appl. Sci., 21 (2011), pp. 1871-1899.

20. J.-L. Lions And E. Magenes, Problèmes aux limites non homogènes et applications, vol. 1 of Travaux et recherches mathématiques, Dunod, Paris, 1968.

21. F. Murat And L. TARTAR, $H$-convergence, in Topics in the mathematical modelling of composite materials, vol. 31 of Progr. Nonlinear Differential Equations Appl., Birkhäuser Boston, Boston, MA, 1997, pp. 21-43.

22. F. Santosa And W. Symes, A dispersive effective medium for wave propagation in periodic composites, SIAM J. Appl. Math., 51 (1991), pp. 984-1005.

23. L. N. Trefethen, Spectral methods in MATLAB, vol. 10, SIAM, 2000. 